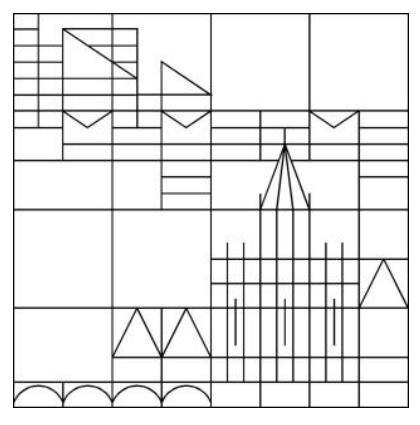

Effects of history and heat models on the stability of thermoelastic Timoshenko systems

\author{
Marcio A. Jorge Silva \\ Reinhard Racke
}

Konstanzer Schriften in Mathematik

Nr. 391, Juni 2020

ISSN 1430-3558

Konstanzer Online-Publikations-System (KOPS)

URL: http://nbn-resolving.de/urn:nbn:de:bsz:352-2-1tfhswxpw76d78 



\title{
Effects of history and heat models on the stability of thermoelastic Timoshenko systems
}

\author{
M. A. Jorge Silva *† \\ Department of Mathematics, State University of Londrina, \\ Londrina 86057-970, PR, Brazil. \\ R. Racke $^{\ddagger}$ \\ Department of Mathematics and Statistics, University of Konstanz, \\ Konstanz 78457, Germany.
}

\begin{abstract}
We investigate different and new thermoelastic Timoshenko systems with or without history, and with Fourier or Cattaneo law for heat conduction, with respect to (non-)exponential stability. Results are obtained that shed a new light on the role of history terms and that of the heat conduction law. Improvements of previous results of earlier work [12] are presented, clarifying open questions, as well as results contrasting [14]. The sensitivity of the Timoshenko system with respect to heat conduction laws and history terms is illustrated.
\end{abstract}

Keywords: Timoshenko system, exponential stability, history, Cattaneo law.

2010 MSC: 35B40, 35Q79, 74F05, 74H40.

\section{Introduction}

The classical conservative Timoshenko system ${ }^{1}$ from [29],

$$
\begin{cases}\rho_{1} \varphi_{t t}-k\left(\varphi_{x}+\psi\right)_{x}=0 & \text { in }(0, L) \times \mathbb{R}^{+} \\ \rho_{2} \psi_{t t}-b \psi_{x x}+k\left(\varphi_{x}+\psi\right)=0 & \text { in }(0, L) \times \mathbb{R}^{+}\end{cases}
$$

for the vertical displacement $\varphi$ and the rotation angle $\psi$ modeling a beam of length $L>0$, has been studied intensively concerning possible damping mechanisms. Two damping terms, one in each equation, of frictional type $\varphi_{t}$ resp. $\psi_{t}$ are easily seen to lead to an exponentially stable system, of course here and in the sequel with added initial and boundary conditions. Only one damping $\psi_{t}$ in the second equation of (1.1) is sufficient for exponential stability if and only if the condition of equality of the wave speeds (EWS), given by

$$
\frac{k}{\rho_{1}}=\frac{b}{\rho_{2}}
$$

*The first author would like to express his gratitude and to thank Prof. R. Racke for all the hospitality during his fruitful scientific visits at the University of Konstanz in the years 2018 and 2019.

${ }^{\dagger}$ Partially supported by Fundação Araucária grant 066/2019 and CNPq grant 301116/2019-9.

${ }^{\ddagger}$ Corresponding author. Email: reinhard.racke@uni-konstanz.de

${ }^{1}$ Recent work strongly suggests the notion of Timoshenko-Ehrenfest system, see [10]. 
is assumed to hold ${ }^{2}$, cf. [27].

Damping through a memory term, replacing the second equation in (1.1),

$$
\begin{cases}\rho_{1} \varphi_{t t}-k\left(\varphi_{x}+\psi\right)_{x}=0 & \text { in }(0, L) \times \mathbb{R}^{+} \\ \rho_{2} \psi_{t t}-b \psi_{x x}+k\left(\varphi_{x}+\psi\right)+\int_{0}^{t} g(t-s) \psi_{x x} d s=0 & \text { in }(0, L) \times \mathbb{R}^{+}\end{cases}
$$

with an exponentially decaying positive kernel $g$, also leads to exponential stability if and only if the EWS condition (1.2) is satisfied, see [6].

Another type of damping consists in taking into account thermal effects - as will be later one main aspect of our paper - having been studied first in [22],

$$
\begin{cases}\rho_{1} \varphi_{t t}-k\left(\varphi_{x}+\psi\right)_{x}=0 & \text { in }(0, L) \times \mathbb{R}^{+}, \\ \rho_{2} \psi_{t t}-b \psi_{x x}+k\left(\varphi_{x}+\psi\right)+\sigma \theta_{x}=0 & \text { in }(0, L) \times \mathbb{R}^{+}, \\ \rho_{3} \theta_{t}-\beta \theta_{x x}+\sigma \psi_{x t}=0 & \text { in }(0, L) \times \mathbb{R}^{+},\end{cases}
$$

where $\theta$ denotes the temperature (difference to a fixed constant reference temperature). Again, exponential stability is given if and only if the EWS condition (1.2) is satisfied. For more recent results related to (1.4) we quote [2] and the references therein.

A third type of damping effect is given by a history term, similar to the memory term in (1.3), resulting in the system

$$
\begin{cases}\rho_{1} \varphi_{t t}-k\left(\varphi_{x}+\psi\right)_{x}=0 & \text { in }(0, L) \times \mathbb{R}^{+} \\ \rho_{2} \psi_{t t}-b \psi_{x x}+k\left(\varphi_{x}+\psi\right)+\int_{0}^{\infty} g(t-s) \psi_{x x} d s=0 & \text { in }(0, L) \times \mathbb{R}^{+}\end{cases}
$$

with an exponentially decaying positive kernel $g$, also becoming exponentially stable if and only if the EWS condition (1.2) is satisfied, see [14, 21]. A short survey of references containing more recent generalized results concerning the models (1.3) and (1.5) can be found in [3].

Combining history and thermal effects, in [14], the thermo-(visco-)elastic system

$$
\begin{cases}\rho_{1} \varphi_{t t}-k\left(\varphi_{x}+\psi\right)_{x}=0 & \text { in }(0, L) \times \mathbb{R}^{+} \\ \rho_{2} \psi_{t t}-b \psi_{x x}+k\left(\varphi_{x}+\psi\right)+\int_{0}^{\infty} g(t-s) \psi_{x x} d s+\sigma \theta_{x}=0 & \text { in }(0, L) \times \mathbb{R}^{+} \\ \rho_{3} \theta_{t}-\beta \theta_{x x}+\sigma \psi_{x t}=0 & \text { in }(0, L) \times \mathbb{R}^{+}\end{cases}
$$

was considered and once more, for exponentially decaying kernels $g$, the exponential stability of the system was obtained if and only if the EWS condition (1.2) is satisfied.

A final aspect in the above mentioned system with temperature and history concerns the behavior, if one replaces the Fourier type heat conduction law

$$
q+\beta \theta_{x}=0
$$

for the heat flux $q$, leading to the classical heat equation visible in its main part

$$
\rho_{3} \theta_{t}-\beta \theta_{x x} \cdots=0
$$

\footnotetext{
${ }^{2}$ The EWS condition is physically never satisfied but demonstrates already the sensitivity of the Timoshenko systems.
} 
above, by the Cattaneo (Maxwell) law

$$
\tau q_{t}+q=-\beta \theta_{x}
$$

with a positive relaxation parameter $\tau>0$ ( $\tau=0$ corresponds to the Fourier law (1.7), combined with the conservation law

$$
\rho_{3} \theta_{t}+q_{x}+\sigma \psi_{x t}=0 .
$$

Here the interesting effect appears that the Cattaneo law leads to non-exponential stability for the system

$$
\begin{cases}\rho_{1} \varphi_{t t}-k\left(\varphi_{x}+\psi\right)_{x}=0 & \text { in }(0, L) \times \mathbb{R}^{+}, \\ \rho_{2} \psi_{t t}-b \psi_{x x}+k\left(\varphi_{x}+\psi\right)+\int_{0}^{\infty} g(t-s) \psi_{x x} d s+\sigma \theta_{x}=0 & \text { in }(0, L) \times \mathbb{R}^{+}, \\ \rho_{3} \theta_{t}+q_{x}+\sigma \psi_{x t}=0 & \text { in }(0, L) \times \mathbb{R}^{+}, \\ \tau q_{t}+q=-\beta \theta_{x}, & \text { in }(0, L) \times \mathbb{R}^{+},\end{cases}
$$

even if the EWS condition (1.2) is satisfied, see again [14]. We also refer to [11] where a new stability number involving the coefficients, based on the development for Cattaneo's system without history [25], is still regarded in the uniform stabilization of (1.9).

Thus one has the surprising fact that the Timoshenko system plus history but without thermal effect, i.e. system (1.5), is exponentially stable under the EWS condition, it remains exponentially stable as expected if we add the (dissipative) effect of heat conduction under the Fourier law in system (1.6), but it loses the exponential stability when adding the (still dissipative?) thermal effect in form of the Cattaneo law in system (1.9). In other words, Cattaneo may destroy exponential stability, while Fourier preserves it. See [14] for detailed (and precise) results on these statements.

In all the thermoelastic models above, the thermal damping is assumed in the bending moment by leading to the couplings in (1.4), (1.6), (1.9).

Here, we shall consider a coupling in the shear moment leading partially to new results with respect to the EWS condition (1.2) - this condition can be avoided under history terms -. In particular, we will have an unexpected very different result (cf. Theorem 4.1), where now we obtain an exponential stability result also under the Cattaneo law.

The thermal damping in the shear force - possibly combined with history in the bending moment - leads to the following thermo-(visco-)elastic system

$$
\begin{cases}\rho_{1} \varphi_{t t}-k\left(\varphi_{x}+\psi\right)_{x}+\sigma \theta_{x}=0 & \text { in }(0, L) \times \mathbb{R}^{+} \\ \rho_{2} \psi_{t t}-b \psi_{x x}+k\left(\varphi_{x}+\psi\right)-\alpha \int_{0}^{\infty} g(s) \psi_{x x}(s) d s-\sigma \theta=0 & \text { in }(0, L) \times \mathbb{R}^{+}, \\ \rho_{3} \theta_{t}+q_{x}+\sigma\left(\varphi_{x}+\psi\right)_{t}=0 & \text { in }(0, L) \times \mathbb{R}^{+}, \\ \tau q_{t}+\beta q+\theta_{x}=0 & \text { in }(0, L) \times \mathbb{R}^{+},\end{cases}
$$

where we have $\alpha=0$ (without history) or $\alpha=1$ (with history), and $\tau=0$ (Fourier law) or $\tau>0$ (Cattaneo law). For the derivation of these possible dissipative hybrid models generated by (1.10), we refer to $[2,3]$ where physical justifications are provided on thermo-(visco-)elasticity.

The remaining constants are assumed to be positive constants,

$$
\rho_{1}, \rho_{2}, \rho_{3}, k, \sigma, b, \beta>0
$$


and further conditions on the exponentially decaying kernel $g$ will be specified later on.

The case $\alpha=0, \tau=0$, meaning without history and with Fourier's law, i.e.

$$
\begin{cases}\rho_{1} \varphi_{t t}-k\left(\varphi_{x}+\psi\right)_{x}+\sigma \theta_{x}=0 & \text { in }(0, L) \times \mathbb{R}^{+}, \\ \rho_{2} \psi_{t t}-b \psi_{x x}+k\left(\varphi_{x}+\psi\right)-\sigma \theta=0 & \text { in }(0, L) \times \mathbb{R}^{+}, \\ \rho_{3} \theta_{t}-\beta \theta_{x x}+\sigma\left(\varphi_{x}+\psi\right)_{t}=0 & \text { in }(0, L) \times \mathbb{R}^{+}\end{cases}
$$

with initial-boundary conditions

$$
\varphi_{x}(0, t)=\varphi_{x}(L, t)=\psi(0, t)=\psi(L, t)=\theta(0, t)=\theta(L, t)=0, \quad t \geq 0,
$$

and

$$
\varphi(x, 0)=\varphi_{0}(x), \varphi_{t}(x, 0)=\varphi_{1}(x), \psi(x, 0)=\psi_{0}(x), \psi_{t}(x, 0)=\psi_{1}(x), \theta(x, 0)=\theta_{0}(x), x \in(0, L),
$$

has already been addressed in the literature, cp. [1, 4, 5]. Accordingly, it is known that (1.12)(1.14) is exponentially stable if and only if the mathematical assumption EWS (1.2) is taken into account, and polynomially stable with optimal decay rate $t^{-1 / 2}$ ([4, Sect. 4]). This result corresponds to the one for system (1.4) (coupling with bending moment, without history, Fourier's law).

Here, we first show in Section 2 the exponential stability if additionally a history term is present, i.e. (1.10) with $\tau=0$ (Fourier law) but $\alpha=1$,

$$
\begin{cases}\rho_{1} \varphi_{t t}-k\left(\varphi_{x}+\psi\right)_{x}+\sigma \theta_{x}=0 & \text { in }(0, L) \times \mathbb{R}^{+}, \\ \rho_{2} \psi_{t t}-b \psi_{x x}+k\left(\varphi_{x}+\psi\right)-\int_{0}^{\infty} g(s) \psi_{x x}(s) d s-\sigma \theta=0 & \text { in }(0, L) \times \mathbb{R}^{+}, \\ \rho_{3} \theta_{t}-\beta \theta_{x x}+\sigma\left(\varphi_{x}+\psi\right)_{t}=0 & \text { in }(0, L) \times \mathbb{R}^{+},\end{cases}
$$

without assuming EWS (1.2), see e.g. Theorem 2.2. This result provides the correct stabilization for (1.15); and essentially improves [12, 28], where the case of non-EWS remained open. It also brings up a different result when compared to (1.6) (thermal coupling on the bending moment) where the EWS condition (1.2) must be regarded for its exponential stabilization. In Section 2 we also provide more precise details on improvements in this case.

In Section 3, we look at Cattaneo's law without history, $\alpha=0$ and $\tau>0$,

$$
\begin{cases}\rho_{1} \varphi_{t t}-k\left(\varphi_{x}+\psi\right)_{x}+\sigma \theta_{x}=0 & \text { in }(0, L) \times \mathbb{R}^{+}, \\ \rho_{2} \psi_{t t}-b \psi_{x x}+k\left(\varphi_{x}+\psi\right)-\sigma \theta=0 & \text { in }(0, L) \times \mathbb{R}^{+}, \\ \rho_{3} \theta_{t}+q_{x}+\sigma\left(\varphi_{x}+\psi\right)_{t}=0 & \text { in }(0, L) \times \mathbb{R}^{+}, \\ \tau q_{t}+\beta q+\theta_{x}=0 & \text { in }(0, L) \times \mathbb{R}^{+}\end{cases}
$$

It will be proved (cf. Theorem 3.1) that there is no exponential stability no matter if EWS (1.2) is true or not. This result is new and corresponds to the known result for the case of damping in the bending moment given in [14].

Finally, we discuss in Section 4 the situation of Cattaneo's law with history, $\alpha=1, \tau>0$, namely,

$$
\begin{cases}\rho_{1} \varphi_{t t}-k\left(\varphi_{x}+\psi\right)_{x}+\sigma \theta_{x}=0 & \text { in }(0, L) \times \mathbb{R}^{+} \\ \rho_{2} \psi_{t t}-\tilde{b} \psi_{x x}+k\left(\varphi_{x}+\psi\right)-\int_{0}^{\infty} g(s) \psi_{x x}(s) d s-\sigma \theta=0 & \text { in }(0, L) \times \mathbb{R}^{+} \\ \rho_{3} \theta_{t}+q_{x}+\sigma\left(\varphi_{x}+\psi\right)_{t}=0 & \text { in }(0, L) \times \mathbb{R}^{+} \\ \tau q_{t}+\beta q+\theta_{x}=0 & \text { in }(0, L) \times \mathbb{R}^{+}\end{cases}
$$


It will be proved that (1.17) is exponentially stable without needing the EWS condition (1.2) on the coefficients, see Theorem 4.1. Unlike the previous case, this is a strong contrast and, maybe, unexpected in comparison to the result for the bending moment damping mentioned above with respect to (1.9), where we lose exponential stability going from the Fourier to the Cattaneo model, as presented in [14]. Sections 3 and 4 will bring all specific and concrete proofs.

As a consequence for the quite different results obtained (in comparison to bending moment damping), quite new sequences of a priori estimates will have to be provided.

Summarizing our contributions, we present:

- a first discussion of several thermoelastic Timoshenko systems involving history terms and both Fourier and Cattaneo models (main Theorems 2.2, 3.1, 4.1);

- new insight into possible roles of history and heat conduction models strongly contrasting expectations from earlier works, in particular Theorems 2.2, 4.1, also answering an open question from $[12,28]$;

- combination of methods requiring new sequences of a priori estimates not given before, under less assumptions on the kernel $g$ as e.g. in [14] (no lower bound required).

We denote by $L^{2}, L^{1}, H^{1}, H_{0}^{1}$ (mainly on the domain $\left.(0, L)\right)$ the usual Sobolev spaces, and by $\langle\cdot, \cdot\rangle_{2}$ and $\|\cdot\|_{2}$ the inner product resp. the norm in $L^{2}$. Unless otherwise specified, the letter $C$ will denote a generic positive constant.

\section{Fourier and history: exponential stability}

We start by considering the thermoelastic Fourier case with history (1.15). Introducing as usual (cf. [14]) for the history setting

$$
\eta(x, t, s):=\psi(x, t)-\psi(x, t-s), \quad t, s \geq 0,
$$

we consider the following system

$$
\begin{cases}\rho_{1} \varphi_{t t}-k\left(\varphi_{x}+\psi\right)_{x}+\sigma \theta_{x}=0 & \text { in }(0, L) \times \mathbb{R}^{+}, \\ \rho_{2} \psi_{t t}-\tilde{b} \psi_{x x}+k\left(\varphi_{x}+\psi\right)-\int_{0}^{\infty} g(s) \eta_{x x}(s) d s-\sigma \theta=0 & \text { in }(0, L) \times \mathbb{R}^{+} \\ \rho_{3} \theta_{t}-\beta \theta_{x x}+\sigma\left(\varphi_{x}+\psi\right)_{t}=0 & \text { in }(0, L) \times \mathbb{R}^{+} \\ \eta_{t}+\eta_{s}-\psi_{t}=0 & \text { in }(0, L) \times \mathbb{R}^{+} \times \mathbb{R}^{+}\end{cases}
$$

where

$$
\tilde{b}:=b-\int_{0}^{\infty} g(s) d s
$$

with initial-boundary conditions

$$
\left\{\begin{array}{l}
\varphi_{x}(0, t)=\varphi_{x}(L, t)=\psi(0, t)=\psi(L, t)=\theta(0, t)=\theta(L, t)=0 \\
\eta(0, t, s)=\eta(L, t, s)=0, \quad t, s \geq 0
\end{array}\right.
$$

and

$$
\left\{\begin{array}{l}
\varphi(x, 0)=\varphi_{0}(x), \varphi_{t}(x, 0)=\varphi_{1}(x), \psi(x, 0)=\psi_{0}(x), \psi_{t}(x, 0)=\psi_{1}(x), \\
\theta(x, 0)=\theta_{0}(x), \eta(x, 0, s)=\eta_{0}(x, s), \eta(x, t, 0)=0, \quad x \in(0, L), t, s \geq 0 .
\end{array}\right.
$$

The assumptions on $g$ are given by 
Assumption 2.1. We assume that $g \in L^{1}\left(\mathbb{R}^{+}\right) \cap C^{1}\left(\mathbb{R}^{+}\right)$is a positive function satisfying

$$
\tilde{b}=b-\int_{0}^{\infty} g(s) d s>0 \quad \text { and } \quad g^{\prime}(s) \leq-k_{1} g(s), s \in \mathbb{R}^{+},
$$

for some constant $k_{1}>0$.

We remark that we do not require any lower bound of type $-k_{0} g(s) \leq g^{\prime}(s)$ or a bound on second derivatives like $\left|g^{\prime \prime}(s)\right| \leq k_{2}$ for some $k_{0}, k_{2}>0$ as in [14]. Instead of using these additional assumptions, we can give an improvement using a technique from [16], see below.

Without the history term it corresponds to system (1.12), where the EWS condition (1.2) is still crucial, see the comments above following (1.12). Now, with history, we will be able to remove this condition and still get exponential stability. This is improves $[12,28]$ answering an open question, and it is in contrast to the corresponding result for systems with thermal damping in the bending moment as in [14], where the EWS condition was also necessary for exponential stability.

To address problem (2.2)-(2.4), we first consider the phase (Hilbert) space

$$
\mathcal{H}_{F}=H_{*}^{1}(0, L) \times L_{*}^{2}(0, L) \times H_{0}^{1}(0, L) \times L^{2}(0, L) \times L^{2}(0, L) \times L_{g}^{2}\left(\mathbb{R}^{+}, H_{0}^{1}(0, L)\right),
$$

where

$$
\begin{aligned}
L_{*}^{2}(0, L):= & \left\{w \in L^{2}(0, L) \mid \int_{0}^{L} w(x) d x=0\right\}, \quad H_{*}^{1}:=H^{1}(0, L) \cap L_{*}^{2}(0, L), \\
& L_{g}^{2}\left(\mathbb{R}^{+}, H_{0}^{1}(0, L)\right):=\left\{w \mid \sqrt{g} w \in L^{2}\left(\mathbb{R}^{+}, H_{0}^{1}(0, L)\right)\right\},
\end{aligned}
$$

endowed with the norm

$$
\|U\|_{\mathcal{H}_{F}}^{2} \equiv \rho_{1}\|\Phi\|_{2}^{2}+\rho_{2}\|\Psi\|_{2}^{2}+k\left\|\varphi_{x}+\psi\right\|_{2}^{2}+\tilde{b}\left\|\psi_{x}\right\|_{2}^{2}+\rho_{3}\|\theta\|_{2}^{2}+\int_{0}^{\infty} g(s)\left\|\eta_{x}(s)\right\|_{2}^{2} d s
$$

and corresponding inner product $\langle\cdot, \cdot\rangle_{\mathcal{H}_{F}}$, for all $U=(\varphi, \Phi, \psi, \Psi, \theta, \eta) \in \mathcal{H}_{F}$. Thus, denoting $\Phi:=\varphi_{t}$ and $\Psi:=\psi_{t}$, we can transform problem (2.2)-(2.4) into the first-order system

$$
\left\{\begin{array}{l}
U_{t}=\mathcal{A}_{F} U, \quad t>0, \\
U(0)=\left(\varphi_{0}, \varphi_{1}, \psi_{0}, \psi_{1}, \theta_{0}, \eta_{0}\right)=: U_{0}
\end{array}\right.
$$

where $\mathcal{A}_{F}: D\left(\mathcal{A}_{F}\right) \subset \mathcal{H}_{F} \rightarrow \mathcal{H}_{F}$ is given by

$$
\mathcal{A}_{F} U:=\left[\begin{array}{c}
\Phi \\
\frac{k}{\rho_{1}}\left(\varphi_{x}+\psi\right)_{x}-\frac{\sigma}{\rho_{1}} \theta_{x} \\
\Psi \\
\frac{1}{\rho_{2}}\left(\tilde{b} \psi+\int_{0}^{\infty} g(s) \eta(s) d s\right)_{x x}-\frac{k}{\rho_{2}}\left(\varphi_{x}+\psi\right)+\frac{\sigma}{\rho_{2}} \theta \\
\frac{\beta}{\rho_{3}} \theta_{x x}-\frac{\sigma}{\rho_{3}}\left(\Phi_{x}+\Psi\right) \\
\Psi-\eta_{s}
\end{array}\right]
$$

with domain

$$
\begin{gathered}
D\left(\mathcal{A}_{F}\right):=\left\{U \in \mathcal{H}_{F} \mid \Phi \in H_{*}^{1}(0, L), \varphi_{x}, \Psi, \theta \in H_{0}^{1}(0, L), \eta_{s} \in L_{g}^{2}\left(\mathbb{R}^{+}, H_{0}^{1}(0, L)\right),\right. \\
\left.\varphi, \theta, \tilde{b} \psi+\int_{0}^{\infty} g(s) \eta(s) d s \in H^{2}(0, L), \eta(\cdot, 0)=0\right\} .
\end{gathered}
$$


It is not difficult to prove that $0 \in \varrho\left(\mathcal{A}_{F}\right)$, with the arguments in [14]. Moreover, $\mathcal{A}_{F}$ is dissipative with

$$
\operatorname{Re}\left(\mathcal{A}_{F} U, U\right)_{\mathcal{H}_{F}}=-\beta\left\|\theta_{x}\right\|_{2}^{2}+\frac{1}{2} \int_{0}^{\infty} g^{\prime}(s)\left\|\eta_{x}(s)\right\|_{2}^{2} d s
$$

This identity would follow easily assuming $-k_{0} g(s) \leq g^{\prime}(s)$ as in [14]. But without this assumption, we can guarantee (2.9) as follows, using arguments given in [16]. The only point to justify is the integration by parts in

$$
-\operatorname{Re} \int_{0}^{\infty} g(s)\left\langle\eta_{s x}(s), \eta_{x}(s)\right\rangle_{2} d s=\frac{1}{2} \int_{0}^{\infty} g^{\prime}(s)\left\|\eta_{x}(s)\right\|_{2}^{2} d s .
$$

Using $\eta_{x}(\cdot, 0)=0$ and denoting by the finite number $\mathrm{Z}$ the left-hand side of $(2.10)$, we have

$$
Z=\lim _{0<y \rightarrow 0}(\underbrace{-g(1 / y)\left\|\eta_{x}(1 / y)\right\|_{2}^{2}}_{=: f_{1}(y)}+\underbrace{\int_{y}^{1 / y} g^{\prime}(s)\left\|\eta_{x}(s)\right\|_{2}^{2} d s}_{=: f_{2}(y)}) .
$$

Since the integrand in $f_{2}(y)$ is negative, $\lim _{0<y \rightarrow 0} f_{2}(y)$ exists and is either a finite negative number or $-\infty$. But the latter is excluded since $f_{1}(y)$ cannot compensate this to a finite number because it is also negative. Hence $f_{1}(y)$ also converges to a finite number which must be zero, otherwise $U=(\ldots, \eta)$ would not belong to the domain of $\mathcal{A}_{F}$. Altogether we have $Z=\lim _{0<y \rightarrow 0} f_{2}(y)$ proving $(2.10)$ and hence $(2.9)$.

Now using Assumption 2.1 we obtain

$$
\begin{aligned}
\operatorname{Re}\left\langle\mathcal{A}_{F} U, U\right\rangle_{\mathcal{H}_{F}} & =-\beta\left\|\theta_{x}\right\|_{2}^{2}+\frac{1}{2} \int_{0}^{\infty} g^{\prime}(s)\left\|\eta_{x}(s)\right\|_{2}^{2} d s \\
& \leq-\beta\left\|\theta_{x}\right\|_{2}^{2}-\frac{k_{1}}{2} \int_{0}^{\infty} g(s)\left\|\eta_{x}(s)\right\|_{2}^{2} d s \\
& \leq 0, \quad \forall U \in D\left(\mathcal{A}_{F}\right) .
\end{aligned}
$$

Therefore, by using the Lumer\&Phillips Theorem, $\mathcal{A}_{F}$ is the infinitesimal generator of a $C_{0}$-semigroup of contractions $\left\{S_{F}(t)\right\}_{t \geq 0}=\left\{e^{\mathcal{A}_{F} t}\right\}_{t \geq 0}$ on $\mathcal{H}_{F}$, and the existence and uniqueness the solution $U(t)=e^{\mathcal{A}_{F} t} U_{0}, t \geq 0$, to problem (2.7) follows in the class

$$
U \in C^{1}\left([0, \infty), \mathcal{H}_{F}\right) \cap C^{0}\left([0, \infty), D\left(\mathcal{A}_{F}\right)\right) .
$$

As main result we have that the semigroup is exponentially stable no matter whether the EWS condition (1.2) is satisfied or not:

Theorem 2.2. Under the Assumption 2.1, there exist constants $C, \gamma>0$, being independent of $U_{0} \in \mathcal{H}_{F}$, such that for all $t \geq 0$

$$
\|U(t)\|_{\mathcal{H}_{F}} \leq C\left\|U_{0}\right\|_{\mathcal{H}_{F}} e^{-\gamma t} .
$$

In other words, the thermo-viscoelastic Timoshenko system under the Fourier law (2.2)-(2.4) is exponentially stable independent of any relation between the coefficients.

To prove Theorem 2.2 we use the well-known characterization of exponential stability for $C_{0}$-semigroups established in $[15,17,26]$, cf. [18]. 
Theorem 2.3. Let $\{T(t)\}_{t \geq 0}=\left\{e^{A t}\right\}_{t \geq 0}$ be a $C_{0}$-semigroup of contractions on a Hilbert space $H$. Then, the semigroup is exponentially if and only if

$$
i \mathbb{R} \subset \varrho(A) \quad \text { (resolvent set) }
$$

and

$$
\limsup _{|\lambda| \rightarrow \infty}\left\|\left(i \lambda I_{d}-A\right)^{-1}\right\|<\infty . \quad \text { (operator norm) }
$$

The conditions (2.13) and (2.14) will be shown in the next subsections.

\subsection{Verifying (2.13): The resolvent set $\varrho\left(\mathcal{A}_{F}\right)$ contains the imaginary axis}

Let us prove that

$$
i \mathbb{R} \subset \varrho\left(\mathcal{A}_{F}\right) .
$$

For this purpose we argue by contradiction, and we suppose that $i \mathbb{R} \not \subset \varrho\left(\mathcal{A}_{F}\right)$. Then, there exist a constant $\omega>0$, w.l.o.g., and a sequence $\lambda_{n} \in \mathbb{R}$, with $0<\lambda_{n} \rightarrow w$ from below and $i \lambda_{n} \in \varrho\left(\mathcal{A}_{F}\right)$, and a sequence of functions

$$
U_{n}=\left(\varphi_{n}, \Phi_{n}, \psi_{n}, \Psi_{n}, \theta_{n}, \eta_{n}\right) \in D\left(\mathcal{A}_{F}\right) \quad \text { with } \quad\left\|U_{n}\right\|_{\mathcal{H}_{F}}=1
$$

such that

$$
i \lambda_{n} U_{n}-\mathcal{A}_{F} U_{n} \rightarrow 0 \quad \text { in } \quad \mathcal{H}_{F} .
$$

Using the expression for $\mathcal{A}_{F}$ given in (2.8), then (2.17) can be rewritten in terms of its components

$$
\begin{cases}i \lambda_{n} \varphi_{n}-\Phi_{n} \rightarrow 0 & \text { in } H_{*}^{1}(0, L), \\ i \lambda_{n} \rho_{1} \Phi_{n}-k\left(\varphi_{n, x}+\psi_{n}\right)_{x}+\sigma \theta_{n, x} \rightarrow 0 & \text { in } L_{*}^{2}(0, L), \\ i \lambda_{n} \psi_{n}-\Psi_{n} \rightarrow 0 & \text { in } H_{0}^{1}(0, L), \\ i \lambda_{n} \rho_{2} \Psi_{n}+k\left(\varphi_{n, x}+\psi_{n}\right) & \end{cases}
$$

Lemma 2.4. Under the assumptions of Theorem 2.2 we have:

$$
\begin{aligned}
& \left\|\theta_{n, x}\right\|_{2}^{2} \rightarrow 0, \text { as } n \rightarrow \infty, \\
& \int_{0}^{\infty}\left[-g^{\prime}(s)\right]\left\|\eta_{n, x}(s)\right\|_{2}^{2} d s \rightarrow 0, \quad \text { as } n \rightarrow \infty \\
& \int_{0}^{\infty} g(s)\left\|\eta_{n, x}(s)\right\|_{2}^{2} d s \rightarrow 0, \text { as } n \rightarrow \infty .
\end{aligned}
$$

Proof. This is an immediate consequence of (2.11) and (2.17). 
Observing Lemma 2.4, the convergence in (2.18) turns into

$$
\begin{cases}i \lambda_{n} \varphi_{n}-\Phi_{n} \rightarrow 0 & \text { in } H_{*}^{1}(0, L), \\ i \lambda_{n} \rho_{1} \Phi_{n}-k\left(\varphi_{n, x}+\psi_{n}\right)_{x} \rightarrow 0 & \text { in } L_{*}^{2}(0, L), \\ i \lambda_{n} \psi_{n}-\Psi_{n} \rightarrow 0 & \text { in } H_{0}^{1}(0, L), \\ i \lambda_{n} \rho_{2} \Psi_{n}+k\left(\varphi_{n, x}+\psi_{n}\right)-\left(\tilde{b} \psi_{n}+\int_{0}^{\infty} g(s) \eta_{n}(s) d s\right)_{x x} \rightarrow 0 & \text { in } L^{2}(0, L), \\ i \lambda_{n} \eta_{n}+\eta_{n, s}-\Psi_{n} \rightarrow 0 & \text { in } L_{g}^{2}\left(\mathbb{R}^{+}, H_{0}^{1}(0, L)\right) .\end{cases}
$$

Lemma 2.5. Under the assumptions of Theorem 2.2 we have:

$$
\left\|\Psi_{n, x}\right\|_{2},\left\|\psi_{n, x}\right\|_{2} \rightarrow 0, \text { as } n \rightarrow \infty .
$$

Proof. First, from (2.22c) one gets

$$
i \lambda_{n}\left\langle\psi_{n, x}, \Psi_{n, x}\right\rangle_{2}-\left\|\Psi_{n, x}\right\|_{2}^{2} \rightarrow 0 .
$$

Using Cauchy-Schwarz and Young inequalities, we get

$$
\left\|\Psi_{n, x}\right\|_{2}^{2} \leq 2\left|i \lambda_{n}\left\langle\psi_{n, x}, \Psi_{n, x}\right\rangle_{2}-\left\|\Psi_{n, x}\right\|_{2}^{2}\right|+\lambda_{n}^{2}\left\|\psi_{n, x}\right\|_{2}^{2} .
$$

Combining (2.24)-(2.25), and since and $\left\|\psi_{n, x}\right\|_{2}^{2} \leq \frac{1}{\beta}\left\|U_{n}\right\|_{\mathcal{H}_{F}}^{2}$, it follows that $\left(\left\|\Psi_{n, x}\right\|_{2}\right)_{n \in \mathbb{N}}$ is bounded.

On the other hand, since $\eta_{n} \in L_{g}^{2}\left(\mathbb{R}^{+}, H_{0}^{1}(0, L)\right)$, we have $g\left\|\eta_{n, x}(\cdot)\right\|_{2}^{2} \in L^{1}\left(\mathbb{R}^{+}\right)$, and

$$
\lim _{z \rightarrow \infty} g(z)\left\|\eta_{n, x}(z)\right\|_{2}^{2}=0
$$

as explained in deriving (2.10).

Now, the mapping $s \mapsto \frac{1}{\lambda_{n}} \Psi_{n} \in L_{g}^{2}\left(\mathbb{R}^{+}, H_{0}^{1}(0, L)\right)$ for all $n \in \mathbb{N}$. Taking the multiplier $\frac{1}{\lambda_{n}^{2}} g(s) \bar{\Psi}_{n}$ in $(2.22 \mathrm{e})$ and taking into account (2.21), we have

$$
\underbrace{\frac{1}{\lambda_{n}^{2}}\left\langle\eta_{n, s}, \Psi_{n}\right\rangle_{L_{g}^{2}\left(\mathbb{R}^{+}, H_{0}^{1}(0, L)\right)}}_{=: P_{n}}-\frac{\int_{0}^{\infty} g(s) d s}{\lambda_{n}^{2}}\left\|\Psi_{n, x}\right\|_{2}^{2} \rightarrow 0 .
$$

Integrating $P_{n}$ by parts with respect to $s$, using Lemma 2.4 (see (2.20)), the fact that $\left(\Psi_{n}\right)_{n \in \mathbb{N}}$ is bounded in $H_{0}^{1}(0, l)$, we infer

$$
\begin{aligned}
\left|P_{n}\right| & \left.=\mid \frac{1}{\lambda_{n}^{2}} \int_{0}^{\infty} g(s)\left\langle\eta_{n, s x}(s), \Psi_{n, x}\right\rangle\right)_{2} d s \mid \\
& =\left|\frac{1}{\lambda_{n}^{2}} \int_{0}^{\infty} g^{\prime}(s)\left\langle\eta_{n, x}(s), \Psi_{n, x}\right\rangle_{2} d s\right| \\
& \leq \frac{1}{\lambda_{n}^{2}}\left\|\Psi_{n, x}\right\|_{2} \|\left.\int_{0}^{\infty} g^{\prime}(s) \eta_{n, x}(s) d s\right|_{2} \\
& \leq \frac{1}{\lambda_{n}^{2}}\left(\int_{0}^{\infty}\left[-g^{\prime}(s)\right] d s\right)^{\frac{1}{2}}\left(\int_{0}^{\infty}\left[-g^{\prime}(s)\right]\left\|\eta_{n, x}(s)\right\|_{2}^{2} d s\right)^{\frac{1}{2}}\left\|\Psi_{n, x}\right\|_{2} \rightarrow 0 .
\end{aligned}
$$

Thus, (2.27) and Lemma 2.4 imply $\Psi_{n} \rightarrow 0$ in $H_{0}^{1}(0, L)$ and, consequently, (2.22c) yields $\psi_{n} \rightarrow 0$ in $H_{0}^{1}(0, L)$ as well. Therefore, the proof of $(2.23)$ is completed. 
Lemma 2.6. Under the assumptions of Theorem 2.2 and the above notations, we have:

$$
\left\|\varphi_{n, x}+\psi_{n}\right\|_{2},\left\|\Phi_{n}\right\|_{2} \rightarrow 0, \text { as } n \rightarrow \infty
$$

Proof. We start by taking the multiplier $k\left(\bar{\varphi}_{n, x}+\bar{\psi}_{n}\right)$ in $(2.22 \mathrm{~d})$ to get

$$
\begin{gathered}
i \lambda_{n} \rho_{2} k\left\langle\Psi_{n}, \varphi_{n, x}+\psi_{n}\right\rangle-\left\langle\left(\beta \psi_{n}+\int_{0}^{\infty} g(s) \eta_{n}(s) d s\right)_{x x}, k\left(\varphi_{n, x}+\psi_{n}\right)\right\rangle_{2} \\
+k^{2}\left\|\varphi_{n, x}+\psi_{n}\right\|_{2}^{2} \rightarrow 0 .
\end{gathered}
$$

Performing integration by parts, using $(2.21),(2.23)$, the boundeness of $\left(\varphi_{n, x}+\psi_{n}\right)_{n \in \mathbb{N}}$ in $L^{2}(0, L)$ and regarding boundary condition, we obtain

$$
k^{2}\left\|\varphi_{n, x}+\psi_{n}\right\|_{2}^{2}+\left\langle\beta \psi_{n, x}+\int_{0}^{\infty} g(s) \eta_{n, x}(s) d s, k\left(\varphi_{n, x}+\psi_{n}\right)_{x}\right\rangle_{2} \rightarrow 0
$$

On the other hand, taking the multiplier $\beta \psi_{n, x}+\int_{0}^{\infty} g(s) \eta_{n, x}(s) d s$ in (2.22b), we have

$$
i \rho_{1} \lambda_{n}\left\langle\beta \psi_{n, x}+\int_{0}^{\infty} g(s) \eta_{n, x}(s) d s, \Phi_{n}\right\rangle_{2}-\left\langle\beta \psi_{n, x}+\int_{0}^{\infty} g(s) \eta_{n, x}(s) d s, k\left(\varphi_{n, x}+\psi_{n}\right)_{x}\right\rangle_{2} \rightarrow 0 .
$$

Using that $\left(\left\|\Phi_{n}\right\|_{2}\right)_{n \in \mathbb{N}}$ is bounded and $\left\|\beta \psi_{n, x}+\int_{0}^{\infty} g(s) \eta_{n, x}(s) d s\right\|_{2} \rightarrow 0$, we conclude from the previous limit that

$$
-\left\langle\beta \psi_{n, x}+\int_{0}^{\infty} g(s) \eta_{n, x}(s) d s, k\left(\varphi_{n, x}+\psi_{n}\right)_{x}\right\rangle_{2} \rightarrow 0
$$

Combining (2.29) and (2.30), we conclude the first converge in (2.28).

Now, taking the multipliers $\rho_{1} \overline{\Phi_{n}}$ in $(2.22 \mathrm{a})$ and $\overline{\varphi_{n}}$ in $(2.22 \mathrm{~b})$ and adding the resulting expressions, we have

$$
i \rho_{1} \lambda_{n}\left[\left\langle\varphi_{n}, \Phi_{n}\right\rangle_{2}+\left\langle\Phi_{n}, \varphi_{n}\right\rangle_{2}\right]-\rho_{1}\left\|\Phi_{n}\right\|_{2}^{2}-k\left\langle\left(\varphi_{n, x}+\psi_{n}\right)_{x}, \varphi_{n}\right\rangle_{2} \rightarrow 0
$$

Integrating by parts and taking the real part, we get

$$
-\rho_{1}\left\|\Phi_{n}\right\|_{2}^{2}+k \operatorname{Re}\left\langle\varphi_{n, x}+\psi_{n}, \varphi_{n, x}\right\rangle_{2} \rightarrow 0 .
$$

On the other hand, taking the multiplier $\rho_{2} \Psi_{n}$ in $(2.22 \mathrm{c})$ and $\overline{\psi_{n}}$ in $(2.22 \mathrm{~d})$, and adding the resulting expressions, we obtain

$$
\begin{aligned}
i \rho_{2} \lambda_{n}\left[\left\langle\psi_{n}, \Psi_{n}\right\rangle_{2}+\left\langle\Psi_{n}, \psi_{n}\right\rangle_{2}\right] & -\left\langle\left(\beta \psi_{n}+\int_{0}^{\infty} g(s) \eta_{n}(s) d s\right)_{x x}, \psi_{n}\right\rangle_{2} \\
& +k\left\langle\varphi_{n, x}+\psi_{n}, \psi_{n}\right\rangle_{2}-\rho_{2}\left\|\Psi_{n}\right\|_{2}^{2} \rightarrow 0 .
\end{aligned}
$$

Integrating by parts, using boundary conditions, and also Lemmas 2.4 and 2.5, we arrive at

$$
k \operatorname{Re}\left\langle\varphi_{n, x}+\psi_{n}, \psi_{n}\right\rangle_{2} \rightarrow 0 .
$$

Adding the limits in (2.31) and (2.32), we get

$$
-\rho_{1}\left\|\Phi_{n}\right\|_{2}^{2}+k\left\|\varphi_{n, x}+\psi_{n}\right\|_{2}^{2} \rightarrow 0,
$$

from where (2.28) follows. Thus the proof of Lemma 2.6 is finished. 
We are finally in the position to give the proof of (2.15). In fact, from (2.19), (2.21), (2.23) and (2.28), we conclude

$$
\left\|U_{n}\right\|_{\mathcal{H}_{F}} \rightarrow 0
$$

which is a contradiction to (2.16).

We remark that, in the proof of (2.15) we use an approach similar to [14, Sect. 4]. However, it is worth mentioning that our refined arguments are different in detail, in particular we do not require a boundedness of the memory kernel from below.

\subsection{Verifying (2.14): Boundedness of $\left(i \lambda I_{d}-\mathcal{A}_{F}\right)^{-1}$}

We will prove that there exists a constant $C>0$ independent of $\lambda \in \mathbb{R}$ such that, as $|\lambda| \rightarrow \infty$,

$$
\left\|\left(i \lambda I_{d}-\mathcal{A}_{F}\right)^{-1}\right\|_{\mathcal{L}\left(\mathcal{H}_{F}\right)} \leq C .
$$

Let $\Upsilon=\left(f_{1}, \ldots, f_{6}\right) \in \mathcal{H}_{F}$ be given, and let

$$
i \lambda U-\mathcal{A}_{F} U=\Upsilon
$$

which in terms of its components is given by

$$
\begin{aligned}
& i \lambda \varphi-\Phi=f_{1}, \\
& i \lambda \rho_{1} \Phi-k\left(\varphi_{x}+\psi\right)_{x}+\sigma \theta_{x}=\rho_{1} f_{2}, \\
& i \lambda \psi-\Psi=f_{3}, \\
& i \lambda \rho_{2} \Psi-\tilde{b} \psi_{x x}-\int_{0}^{\infty} g(s) \eta_{x x}(s) d s+k\left(\varphi_{x}+\psi\right)-\sigma \theta=\rho_{2} f_{4}, \\
& i \lambda \rho_{3} \theta-\beta \theta_{x x}+\sigma\left(\Phi_{x}+\Psi\right)=\rho_{3} f_{5}, \\
& i \lambda \eta+\eta_{s}-\Psi=f_{6} .
\end{aligned}
$$

To prove (2.33) we have to show that there exists a constant $C>0$, independent of $\lambda$, such that, as $|\lambda| \rightarrow \infty$,

$$
\|U\|_{\mathcal{H}_{F}} \leq C\|\Upsilon\|_{\mathcal{H}_{F}} .
$$

The estimate (2.36) will be proved in different steps estimating the different components.

Lemma 2.7. Under the assumptions of Theorem 2.2, there exists a constant $C>0$ independent of $\lambda$ such that

$$
\left\|\theta_{x}\right\|_{2}^{2}, \int_{0}^{\infty}\left[-g^{\prime}(s)\right]\left\|\eta_{x}(s)\right\|_{2}^{2} d s \leq C\|U\|_{\mathcal{H}_{F}}\|\Upsilon\|_{\mathcal{H}_{F}} .
$$

In particular, there exists a constant $C>0$, independent of $\lambda$, such that

$$
\rho_{3}\|\theta\|_{2}^{2}+\int_{0}^{\infty} g(s)\left\|\eta_{x}(s)\right\|_{2}^{2} d s \leq C\|U\|_{\mathcal{H}_{F}}\|\Upsilon\|_{\mathcal{H}_{F}} .
$$

Proof. Estimate (2.37) follows immediately by taking the inner product of (2.34) with $U$ in $\mathcal{H}_{F}$ and using (2.11).

Lemma 2.8. Under the assumptions of Theorem 2.2 and given any $\epsilon>0$, there exists a constant $C_{\epsilon}>0$, independent of $\lambda$, such that

$$
k\left\|\varphi_{x}+\psi\right\|_{2}^{2} \leq \epsilon\|U\|_{\mathcal{H}_{F}}^{2}+C_{\epsilon}\|\Upsilon\|_{\mathcal{H}_{F}}^{2},
$$

for $|\lambda|>1$ large enough. 
Proof. From the resolvent equations (2.35a), (2.35c) and (2.35e), we have

$$
i \lambda \rho_{3} \theta-\beta \theta_{x x}+i \lambda \sigma\left(\varphi_{x}+\psi\right)=\rho_{3} f_{5}+\sigma\left(f_{1, x}+f_{3}\right) .
$$

Multiplying (2.40) by $k \overline{\left(\varphi_{x}+\psi\right)}$ and integrating over $(0, L)$, we infer

$$
\begin{aligned}
i \lambda \sigma k \int_{0}^{L}\left|\varphi_{x}+\psi\right|^{2} d x= & \underbrace{-\beta \int_{0}^{L} \theta_{x} \overline{\left[k\left(\varphi_{x}+\psi\right)_{x}\right]} d x}_{=: R_{1}}+\underbrace{\rho_{3} k \int_{0}^{L} \theta \overline{\int_{0}\left(i \lambda\left(\varphi_{x}+\psi\right)\right]} d x}_{=: R_{2}} \\
& +k \int_{0}^{L}\left[\rho_{3} f_{5}+\sigma\left(f_{1, x}+f_{3}\right)\right] \overline{\left(\varphi_{x}+\psi\right)} d x .
\end{aligned}
$$

Let us rewrite the terms $R_{1}$ and $R_{2}$ as follows. First, using (2.35b) we get

$$
R_{1}=i \lambda \beta \rho_{1} \int_{0}^{L} \theta_{x} \bar{\Phi} d x-\beta \sigma \int_{0}^{L}\left|\theta_{x}\right|^{2} d x+\beta \rho_{1} \int_{0}^{L} \theta_{x} \overline{f_{2}} d x .
$$

Next, applying (2.35a), (2.35c), and integration by parts, we obtain

$$
R_{2}=-k \rho_{3} \int_{0}^{L} \theta_{x} \bar{\Phi} d x+k \rho_{3} \int_{0}^{L} \theta \bar{\Psi} d x+k \rho_{3} \int_{0}^{L} \theta \overline{\left(f_{1, x}+f_{3}\right)} d x .
$$

Replacing the above expressions for $R_{1}$ and $R_{2}$ in (2.41), and denoting by

$$
\begin{aligned}
R_{3}:= & -\beta \sigma \int_{0}^{L}\left|\theta_{x}\right|^{2} d x-k \rho_{3} \int_{0}^{L} \theta_{x} \bar{\Phi} d x+k \rho_{3} \int_{0}^{L} \theta \bar{\Psi} d x \\
& +k \rho_{3} \int_{0}^{L} \theta \overline{\left(f_{1, x}+f_{3}\right)} d x+\beta \rho_{1} \int_{0}^{L} \theta_{x} \overline{f_{2}} d x \\
& +k \int_{0}^{L}\left[\rho_{3} f_{5}+\sigma\left(f_{1, x}+f_{3}\right)\right] \overline{\left(\varphi_{x}+\psi\right)} d x
\end{aligned}
$$

it follows that

$$
i \lambda \sigma k\left\|\varphi_{x}+\psi\right\|_{2}^{2}=i \lambda \beta \rho_{1} \int_{0}^{L} \theta_{x} \bar{\Phi} d x+R_{3}
$$

Now, from the estimate (2.37) and Poincaré's inequality there exists a constant $C>0$ such that

$$
\left|R_{3}\right| \leq C\|U\|_{\mathcal{H}_{F}}\|\Upsilon\|_{\mathcal{H}_{F}}+C\left\|\theta_{x}\right\|_{2}\|U\|_{\mathcal{H}_{F}}+C\left\|\theta_{x}\right\|_{2}\|\Upsilon\|_{\mathcal{H}_{F}}
$$

Returning to the identity (2.42), one sees that

$$
\begin{aligned}
k\left\|\varphi_{x}+\psi\right\|_{2}^{2} \leq & \frac{\beta \rho_{1}}{\sigma}\left\|\theta_{x}\right\|_{2}\|\Phi\|_{2}+\frac{1}{\sigma|\lambda|}\left|R_{3}\right| \\
\leq & C\left\|\theta_{x}\right\|_{2}\|\Phi\|_{2}+\frac{C}{|\lambda|}\left\|\theta_{x}\right\|_{2}\|U\|_{\mathcal{H}_{F}} \\
& +\frac{C}{|\lambda|}\|U\|_{\mathcal{H}_{F}}\|\Upsilon\|_{\mathcal{H}_{F}}+\frac{C}{|\lambda|}\left\|\theta_{x}\right\|_{2}\|\Upsilon\|_{\mathcal{H}_{F}} .
\end{aligned}
$$

From (2.43), using again the previous estimate (2.37) and Young's inequality with $\epsilon>0$ several times, we conclude (2.39) for $|\lambda|>1$. 
Lemma 2.9. Under the assumptions of Theorem 2.2 and given any $\epsilon>0$, there exists a constant $C_{\epsilon}>0$, independent of $\lambda$, such that

$$
\rho_{1}\|\Phi\|_{2}^{2} \leq \epsilon\|U\|_{\mathcal{H}_{F}}^{2}+C_{\epsilon}\|\Upsilon\|_{\mathcal{H}_{F}}^{2}
$$

for $|\lambda|>1$ large enough.

Proof. Multiplying (2.35b) by $-\bar{\varphi}$, integrating on $(0, L)$ and observing $(2.35 \mathrm{a})$, we get

$$
\rho_{1} \int_{0}^{L}|\Phi|^{2} d x=k \int_{0}^{L}\left|\varphi_{x}+\psi\right|^{2} d x-k \int_{0}^{L}\left(\varphi_{x}+\psi\right) \bar{\psi} d x+R_{4}
$$

where we have added and subtracted the term $k \int_{0}^{L}\left(\varphi_{x}+\psi\right) \bar{\psi} d x$ and denoted

$$
R_{4}:=\frac{i}{\lambda} \sigma \int_{0}^{L} \theta_{x} \overline{\left(\Phi+f_{1}\right)} d x-\rho_{1} \int_{0}^{L}\left(\Phi \overline{f_{1}}+f_{2} \bar{\varphi}\right) d x .
$$

Obviously we have

$$
\left|R_{4}\right| \leq \frac{C}{|\lambda|}\left\|\theta_{x}\right\|_{2}\|U\|_{\mathcal{H}_{F}}+\frac{C}{|\lambda|}\left\|\theta_{x}\right\|_{2}\|\Upsilon\|_{\mathcal{H}_{F}}+C\|U\|_{\mathcal{H}_{F}}\|\Upsilon\|_{\mathcal{H}_{F}}
$$

Then, going back to (2.45), using this latter estimate, the resolvent equation $(2.35 \mathrm{c})$ and also (2.43) along with proper Young inequalities, one has

$$
\begin{aligned}
\rho_{1}\|\Phi\|_{2}^{2} \leq & k\left\|\varphi_{x}+\psi\right\|_{2}^{2}+k\left\|\varphi_{x}+\psi\right\|_{2}\|\psi\|_{2}+\left|R_{4}\right| \\
\leq & C\left\|\varphi_{x}+\psi\right\|_{2}^{2}+\frac{C}{|\lambda|}\left\|\theta_{x}\right\|_{2}\|U\|_{\mathcal{H}_{F}}+\frac{C}{|\lambda|}\left\|\theta_{x}\right\|_{2}\|\Upsilon\|_{\mathcal{H}_{F}} \\
& +C\|U\|_{\mathcal{H}_{F}}\|\Upsilon\|_{\mathcal{H}_{F}}+\frac{C}{|\lambda|}\|U\|_{\mathcal{H}_{F}}^{2}+C\|\Upsilon\|_{\mathcal{H}_{F}}^{2},
\end{aligned}
$$

for $|\lambda|>1$ and some constant $C>0$. Using again the estimates (2.43), (2.37), and Young's inequality with $\epsilon>0$ several times, we conclude (2.44) for $|\lambda|>1$ large enough.

Lemma 2.10. Under the assumptions of Theorem 2.2 and given any $\epsilon>0$, there exists a constant $C_{\epsilon}>0$, independent of $\lambda$, such that

$$
\rho_{2}\|\Psi\|_{2}^{2} \leq \epsilon\|U\|_{\mathcal{H}_{F}}^{2}+C_{\epsilon}\|\Upsilon\|_{\mathcal{H}_{F}}^{2}
$$

for $|\lambda|>1$ large enough.

Proof. Multiplying (2.35d) by $\int_{0}^{\infty} g(s) \overline{\eta(s)} d s$ and integrating over $(0, L)$, we get

$$
\begin{gathered}
\underbrace{\left.-\rho_{2} \int_{0}^{L} \int_{0}^{\infty} g(s) \Psi \overline{[i \lambda \eta(s)}\right] d s d x}_{=: R_{5}}-\sigma \int_{0}^{L} \int_{0}^{\infty} g(s) \overline{\theta \eta(s)} d s d x+\tilde{b} \int_{0}^{L} \int_{0}^{\infty} g(s) \overline{\eta_{x}(s)} \psi_{x} d s d x \\
+\int_{0}^{L}\left|\int_{0}^{\infty} g(s) \eta_{x}(s) d s\right|^{2} d x+\underbrace{k \int_{0}^{L} \int_{0}^{\infty} g(s)\left(\varphi_{x}+\psi\right) \overline{\eta(s)} d s d x}_{=: R_{6}}=\rho_{2} \int_{0}^{L} \int_{0}^{\infty} g(s) f_{4} \overline{\eta(s)} d s d x .
\end{gathered}
$$


Now, using the identity (2.35f) in $R_{5}$ and the expressions (2.35a) and (2.35c) in $R_{6}$, results in

$$
\begin{aligned}
-\overbrace{2} \overbrace{\left(\int_{0}^{\infty} g(s) d s\right)}^{=: b_{0}} \int_{0}^{L}|\Psi|^{2} d x= & \sigma \int_{0}^{L} \int_{0}^{\infty} g(s) \theta \overline{\eta(s)} d s d x-\tilde{b} \int_{0}^{L} \int_{0}^{\infty} g(s) \overline{\eta_{x}(s)} \psi_{x} d s d x \\
& +\rho_{2} \int_{0}^{L} \int_{0}^{\infty} g(s) f_{4} \overline{\eta(s)} d s d x+\rho_{2} \int_{0}^{L} \int_{0}^{\infty} g(s) \Psi \overline{f_{6}} d s d x \\
& +R_{7}+R_{8}+R_{9}
\end{aligned}
$$

where we denote

$$
R_{7}:=-\rho_{2} \int_{0}^{L} \int_{0}^{\infty} g(s) \Psi \overline{\left[\eta_{s}(s)\right]} d s d x, \quad R_{8}:=-\int_{0}^{L}\left(\int_{0}^{\infty} g(s) \eta_{x}(s) d s\right)^{2} d x
$$

and

$$
\begin{aligned}
R_{9}:= & -\frac{i k}{\lambda} \int_{0}^{L} \int_{0}^{\infty} g(s) \overline{\eta_{x}(s)} d s \Phi d x+\frac{i k}{\lambda} \int_{0}^{L} \int_{0}^{\infty} g(s) \overline{\eta(s)} d s \Psi d x \\
& +\frac{i k}{\lambda} \int_{0}^{L} \int_{0}^{\infty} g(s) \overline{\eta(s)} d s\left(f_{1, x}+f_{3}\right) d x .
\end{aligned}
$$

We obtain for $R_{7}, R_{8}$ and $R_{9}$ :

$$
\left|R_{7}\right| \leq \rho_{2} b_{0}{ }^{1 / 2}\|\Psi\|_{2}\left(\int_{0}^{\infty}\left[-g^{\prime}(s)\right]\|\eta(s)\|_{2}^{2} d s\right)^{1 / 2}
$$

Next,

$$
\left|R_{8}\right| \leq b_{0}\|\eta\|_{L_{g}^{2}}^{2}
$$

remembering the notation $\|\eta\|_{L_{g}^{2}}^{2}=\int_{0}^{\infty} g(s)\left\|\eta_{x}(s)\right\|_{2}^{2} d s$. Moreover, there exists a constant $C>0$ such that

$$
\left|R_{9}\right| \leq \frac{C}{|\lambda|}\|\eta\|_{L_{g}^{2}}\|\Phi\|_{2}+\frac{C}{|\lambda|}\|\eta\|_{L_{g}^{2}}\|\Psi\|_{2}+\frac{C}{|\lambda|}\|\eta\|_{L_{g}^{2}}\left\|f_{1, x}+f_{3}\right\|_{2} .
$$

Using these last three estimates in (2.47) and also (2.38), we arrive at

$$
\begin{aligned}
\rho_{2}\|\Psi\|_{2}^{2} \leq & C\|\eta\|_{L_{g}^{2}}\|\theta\|_{2}+C\|\eta\|_{L_{g}^{2}}\left\|\psi_{x}\right\|_{2}+C\|U\|_{\mathcal{H}_{F}}\|\Upsilon\|_{\mathcal{H}_{F}} \\
& +C\|\Psi\|_{2}\left(\int_{0}^{\infty}\left[-g^{\prime}(s)\right]\left\|\eta_{x}(s)\right\|_{2}^{2} d s\right)^{1 / 2} \\
& +\frac{C}{|\lambda|}\|\eta\|_{L_{g}^{2}}\|\Phi\|_{2}+\frac{C}{|\lambda|}\|\eta\|_{L_{g}^{2}}\|\Psi\|_{2},
\end{aligned}
$$

for come constant $C>0$ and $|\lambda|>1$. From (2.37) and (2.38) we deduce

$$
\rho_{2}\|\Psi\|_{2}^{2} \leq C\|U\|_{\mathcal{H}_{F}}\|\Upsilon\|_{\mathcal{H}_{F}}+C\|\eta\|_{L_{g}^{2}}\left\|\psi_{x}\right\|_{2}+\frac{C}{|\lambda|}\|\eta\|_{L_{g}^{2}}\|\Phi\|_{2},
$$

for come constant $C>0$ and $|\lambda|>1$. Finally, from (2.49), using again the estimate (2.38), we conclude (2.46) for $|\lambda|>1$ large enough. 
Lemma 2.11. Under the assumptions of Theorem 2.2 and given any $\epsilon>0$, there exists a constant $C_{\epsilon}>0$, independent of $\lambda$, such that

$$
\tilde{b}\left\|\psi_{x}\right\|_{2}^{2} \leq \epsilon\|U\|_{\mathcal{H}_{F}}^{2}+C_{\epsilon}\|\Upsilon\|_{\mathcal{H}_{F}}^{2},
$$

for $|\lambda|>1$ large enough.

Proof. Multiplying $(2.35 \mathrm{~d})$ by $\bar{\psi}$ and integrating on $(0, L)$, we have

$$
\begin{gathered}
\underbrace{-\rho_{2} \int_{0}^{L} \Psi \overline{(i \lambda \psi)} d x}_{=: R_{10}}+\tilde{b} \int_{0}^{L}\left|\psi_{x}\right|^{2} d x+\int_{0}^{L} \int_{0}^{\infty} g(s) \eta_{x}(s) \overline{\psi_{x}} d s d x \\
+\underbrace{k \int_{0}^{L}\left(\varphi_{x}+\psi\right) \bar{\psi} d x}_{=: R_{11}}-\sigma \int_{0}^{L} \theta \bar{\psi} d x=\rho_{2} \int_{0}^{L} f_{4} \bar{\psi} d x .
\end{gathered}
$$

Replacing $\psi$ given by the resolvent equation (2.35c) in both $R_{10}$ and $R_{11}$, one has

$$
\begin{aligned}
\tilde{b} \int_{0}^{L}\left|\psi_{x}\right|^{2} d x= & -\int_{0}^{L} \int_{0}^{\infty} g(s) \eta_{x}(s) \overline{\psi_{x}} d s d x+\frac{i k}{\lambda} \int_{0}^{L}\left(\varphi_{x}+\psi\right) \bar{\Psi} d x \\
& +\sigma \int_{0}^{L} \theta \bar{\psi} d x+\rho_{2} \int_{0}^{L}|\Psi|^{2} d x+R_{12}
\end{aligned}
$$

where

$$
R_{12}:=\frac{i k}{\lambda} \int_{0}^{L}\left(\varphi_{x}+\psi\right) \overline{f_{3}} d x+\rho_{2} \int_{0}^{L} f_{4} \bar{\psi} d x+\rho_{2} \int_{0}^{L} \Psi \overline{f_{3}} d x .
$$

It is easy to see that

$$
\left|R_{12}\right| \leq C\|U\|_{\mathcal{H}_{F}}\|\Upsilon\|_{\mathcal{H}_{F}},
$$

for some constant $C>0$, if $|\lambda|>1$. Thus, using (2.43) and (2.49), we obtain from (2.51)

$$
\tilde{b}\left\|\psi_{x}\right\|_{2}^{2} \leq C\|U\|_{\mathcal{H}_{F}}\|\Upsilon\|_{\mathcal{H}_{F}}+C\|\eta\|_{L_{g}^{2}}\|U\|_{\mathcal{H}_{F}}+C\left\|\theta_{x}\right\|_{2}\|U\|_{\mathcal{H}_{F}}+\left\|\theta_{x}\right\|_{2}\|\Upsilon\|_{\mathcal{H}_{F}}
$$

for come constant $C>0$ and $|\lambda|>1$. Last, observing the useful estimates (2.37)-(2.38), we finally conclude $(2.50)$ for $|\lambda|>1$.

Finally, taking into account the Lemmas 2.7-2.11 and choosing $\epsilon>0$ small enough, there exists a constant $C>0$ independent of $\lambda$ such that (2.36) holds true.

This completes the proof of Theorem 2.2.

Remark 2.12. Let us finish this section with some comments as follows.

1. Theorem 2.2 is addressed for the mixed Dirichlet-Neumann boundary condition (2.3) only, but the same result holds true for other different boundary conditions as well, such as the Dirichlet-Dirichlet ones

$$
\varphi(x, t)=\psi(x, t)=\theta(x, t)=\eta^{t}(x, s)=0 \quad \text { for } \quad x=0, L, t, s \geq 0 .
$$

Indeed, for the latter and its proper spaces for solutions, we still follow the same spirit of computations as done in the proof of Theorem 2.2, by noting that the only difference comes from the (possible) point-wise boundary terms. However, to handle with them we 
can use the same point-wise estimates as provided in [1,21] or else introduce useful cut-off functions and work with local estimates instead, as considered e.g. in [4, 5], and then extend the estimate to the whole range $(0, L)$ by means of an observability analysis for Timoshenko systems. In conclusion, problem (2.2)-(2.4) is also exponential stable subject to any other different boundary condition instead of (2.3) where well-posedness is ensured.

2. Theorem 2.2 gives the answer to a question raised in [28, Rem. 3.8] with respect to thermoviscoelastic Timoshenko systems under Fourier's law and memory in a history setting, improving significantly some results presented in [12] (see Theorems 2.2 and 2.3 therein), once Theorem 2.2 has revealed that the uniform exponential stability is achieved with no necessity of the EWS assumption (1.2) nor higher regularity of initial data. Moreover, even for memory with null history the result on exponential stability keeps unchanged for (2.2)-(2.4), including boundary condition (2.52), and the proof could be done through perturbed energy method by combing similar arguments as given in [21, 22] and refining the computations of [7, 19, 20] in the case where the function $\xi$ therein is constant. Therefore, the exponential stability result correspondingly also holds for Timoshenko problems with null history and Fourier's law under exponential memory kernels without regarding EWS, which gives a different view of the stability result in [20, Thm. 2.5] for exponential kernels, i.e. $\xi(\cdot) \equiv \xi$ constant in [20]. It also complements the statements in [7, Rem. 3.4] since the shear thermal coupling is the responsible for neutralizing the requirement of EWS assumption, not the Neumann condition considered in [7] (without EWS) in comparison to the Dirichlet conditions (2.52) in [20] (with EWS).

\section{Cattaneo without history: non-exponential stability}

In this section we consider the model (1.16), with Cattaneo type heat conduction and without a history term,

$$
\begin{cases}\rho_{1} \varphi_{t t}-k\left(\varphi_{x}+\psi\right)_{x}+\sigma \theta_{x}=0 & \text { in }(0, L) \times \mathbb{R}^{+}, \\ \rho_{2} \psi_{t t}-b \psi_{x x}+k\left(\varphi_{x}+\psi\right)-\sigma \theta=0 & \text { in }(0, L) \times \mathbb{R}^{+}, \\ \rho_{3} \theta_{t}+q_{x}+\sigma\left(\varphi_{x}+\psi\right)_{t}=0 & \text { in }(0, L) \times \mathbb{R}^{+}, \\ \tau q_{t}+\beta q+\theta_{x}=0 & \text { in }(0, L) \times \mathbb{R}^{+},\end{cases}
$$

where $\tau>0$, and with initial-boundary conditions

$$
\varphi_{x}(0, t)=\varphi_{x}(L, t)=\psi(0, t)=\psi(L, t)=\theta(0, t)=\theta(L, t)=0, \quad t \geq 0,
$$

and

$$
\left\{\begin{array}{l}
\varphi(x, 0)=\varphi_{0}(x), \varphi_{t}(x, 0)=\varphi_{1}(x), \psi(x, 0)=\psi_{0}(x), \psi_{t}(x, 0)=\psi_{1}(x), \\
\theta(x, 0)=\theta_{0}(x), q(x, 0)=q_{0}(x), \quad x \in(0, L) .
\end{array}\right.
$$

The corresponding system with Fourier type heat conduction $(\tau=0)$ is exponentially stable if and only if the EWS condition (1.2) is satisfied, as explained in the Introduction. Now we shall see that the system above is not exponentially stable even if the EWS condition is satisfied. That means, the system loses the property of being exponentially stable when taking Cattaneo's instead of Fourier's law. This corresponds to the situation of thermal damping in the bending moment considered in [14], while we have the damping in the shear moment.

To address problem (3.1)-(3.3), we consider the phase (Hilbert) space

$$
\mathcal{H}_{C_{1}}:=H_{*}^{1}(0, L) \times L_{*}^{2}(0, L) \times H_{0}^{1}(0, L) \times L^{2}(0, L) \times L^{2}(0, L) \times L^{2}(0, L),
$$


equipped with the norm

$$
\|U\|_{\mathcal{H}_{C_{1}}}^{2} \equiv \rho_{1}\|\Phi\|_{2}^{2}+\rho_{2}\|\Psi\|_{2}^{2}+k\left\|\varphi_{x}+\psi\right\|_{2}^{2}+b\left\|\psi_{x}\right\|_{2}^{2}+\rho_{3}\|\theta\|_{2}^{2}+\tau\|q\|_{2}^{2}
$$

for $U=(\varphi, \Phi, \psi, \Psi, \theta, q) \in \mathcal{H}_{C_{1}}$. Thus, denoting $\Phi=\varphi_{t}$ and $\Psi=\psi_{t}$, we can transform problem (3.1)-(3.3) into the first-order system

$$
\left\{\begin{array}{l}
U_{t}=\mathcal{A}_{C_{1}} U, \quad t>0, \\
U(0)=\left(\varphi_{0}, \varphi_{1}, \psi_{0}, \psi_{1}, \theta_{0}, q_{0}\right)=: U_{0},
\end{array}\right.
$$

where $\mathcal{A}_{C_{1}}: D\left(\mathcal{A}_{C_{1}}\right) \subset \mathcal{H}_{C_{1}} \rightarrow \mathcal{H}_{C_{1}}$ is given by

$$
\mathcal{A}_{C_{1}} U:=\left[\begin{array}{c}
\Phi \\
\frac{k}{\rho_{1}}\left(\varphi_{x}+\psi\right)_{x}-\frac{\sigma}{\rho_{1}} \theta_{x} \\
\Psi \\
\frac{b}{\rho_{2}} \psi_{x x}-\frac{k}{\rho_{2}}\left(\varphi_{x}+\psi\right)+\frac{\sigma}{\rho_{2}} \theta \\
-\frac{1}{\rho_{3}} q_{x}-\frac{\sigma}{\rho_{3}}\left(\Phi_{x}+\Psi\right) \\
-\frac{\beta}{\tau} q-\frac{1}{\tau} \theta_{x}
\end{array}\right]
$$

with domain

$$
D\left(\mathcal{A}_{C_{1}}\right):=\left\{U \in \mathcal{H}_{C_{1}} \mid \Phi \in H_{*}^{1}, \varphi_{x}, \Psi, \theta \in H_{0}^{1}, q \in H^{1}, \varphi, \psi \in H^{2}\right\} .
$$

It is not difficult to prove that $0 \in \varrho\left(\mathcal{A}_{C_{1}}\right)$ and that $\mathcal{A}_{C_{1}}$ is dissipative,

$$
\operatorname{Re}\left\langle\mathcal{A}_{C_{1}} U, U\right\rangle_{\mathcal{H}_{C_{1}}}=-\beta\|q\|_{2}^{2} \leq 0 .
$$

Therefore, using the Lumer-Phillips Theorem again, $\mathcal{A}_{C_{1}}$ is the infinitesimal generator of a $C_{0^{-}}$ semigroup of contractions $\left\{S_{C_{1}}(t)\right\}_{t \geq 0}=\left\{e^{\mathcal{A}_{C_{1}} t}\right\}_{t \geq 0}$ on $\mathcal{H}_{C_{1}}$, and the existence and uniqueness of solutions to problem (3.5) follows.

We are going to show that the semigroup is not exponentially stable even assuming condition $(1.2)$.

Theorem 3.1. The $C_{0}$-semigroup of contractions $\left\{S_{C_{1}}(t)\right\}_{t \geq 0}$ is not exponentially stable. In other words, the thermoelastic Timoshenko system under the Cattaneo law without history, (3.1)(3.3), is not exponentially stable, whether condition (1.2) holds or not.

Proof. We start by noting that it is relatively simple prove that $D\left(\mathcal{A}_{C_{1}}\right)$ is compactly embedded into $\mathcal{H}_{C_{1}}$, and also that the operator $i \lambda I_{d}-\mathcal{A}_{C_{1}}$ is injective for any given $\lambda \in \mathbb{R}$. Therefore, one can conclude that $i \mathbb{R} \subset \rho\left(\mathcal{A}_{C_{1}}\right)$. That is, condition (2.13) in Theorem 2.3 is satisfied. Consequently, semigroup converges strongly (i.e. for any fixed initial value) to zero, see e.g. [8, 9]. But, as we are going to see below, the second necessary condition for exponential stability (2.14) is no longer valid. To this end, it is enough to show the existence of a sequence $\left(\lambda_{n}\right)_{n \in \mathbb{N}} \subset \mathbb{R}$, with $\left|\lambda_{n}\right| \rightarrow \infty$, and $U_{n} \in D\left(\mathcal{A}_{C_{1}}\right), n \in \mathbb{N}$, such that

$$
\lim _{n \rightarrow \infty}\left\|U_{n}\right\|_{\mathcal{H}_{c}} \equiv \lim _{n \rightarrow \infty}\left\|\left(i \lambda_{n} I_{d}-\mathcal{A}_{C_{1}}\right)^{-1} F_{n}\right\|_{\mathcal{H}_{c}}=\infty
$$


for some bounded sequence $\left(F_{n}\right)_{n \in \mathbb{N}} \subset \mathcal{H}_{C_{1}}$ bounded. Indeed, let us consider

$$
F_{n}:=\left(0,0,0, \sin \left(\alpha \lambda_{n} x\right), 0,0\right) \quad \text { with } \quad \alpha:=\sqrt{\frac{\rho_{2}}{b}}, \lambda_{n}:=\frac{n \pi}{\alpha L}, n \in \mathbb{N}
$$

In order to simplify the notations, let us omit the index $n$ in the sequel. Let $U \equiv(\varphi, \Phi, \psi, \Psi, \theta, q)$ be the solution of the resolvent equation $\left(i \lambda I_{d}-\mathcal{A}_{C_{1}}\right) U=F$, which in terms of its components reads

$$
\left\{\begin{array}{l}
i \lambda \varphi-\Phi=0 \\
i \lambda \Phi-\frac{k}{\rho_{1}}\left(\varphi_{x}+\psi\right)_{x}+\frac{\sigma}{\rho_{1}} \theta_{x}=0 \\
i \lambda \psi-\Psi=0 \\
i \lambda \Psi-\frac{b}{\rho_{2}} \psi_{x x}+\frac{k}{\rho_{2}}\left(\varphi_{x}+\psi\right)-\frac{\sigma}{\rho_{2}} \theta=\sin (\alpha \lambda x), \\
i \lambda \theta+\frac{1}{\rho_{3}} q_{x}+\frac{\sigma}{\rho_{3}}\left(\Phi_{x}+\Psi\right)=0 \\
i \lambda q+\frac{\beta}{\tau} q+\frac{1}{\tau} \theta_{x}=0
\end{array}\right.
$$

From $(3.9)_{1}$ and $(3.9)_{3}$ we get the following reduced system

$$
\left\{\begin{array}{l}
-\lambda^{2} \varphi-\frac{k}{\rho_{1}}\left(\varphi_{x}+\psi\right)_{x}+\frac{\sigma}{\rho_{1}} \theta_{x}=0 \\
-\lambda^{2} \psi-\frac{b}{\rho_{2}} \psi_{x x}+\frac{k}{\rho_{2}}\left(\varphi_{x}+\psi\right)-\frac{\sigma}{\rho_{2}} \theta=\sin (\alpha \lambda x), \\
i \lambda \theta+\frac{1}{\rho_{3}} q_{x}+\frac{i \lambda \sigma}{\rho_{3}}\left(\varphi_{x}+\psi\right)=0 \\
i \lambda q+\frac{\beta}{\tau} q+\frac{1}{\tau} \theta_{x}=0 .
\end{array}\right.
$$

Now, by virtue of the boundary conditions in (3.2), we look for solutions of (3.10) given by

$$
\varphi(x)=A \cos (\alpha \lambda x), \psi(x)=B \sin (\alpha \lambda x), \theta(x)=C \sin (\alpha \lambda x), q(x)=D \cos (\alpha \lambda x),
$$

where $A=A_{\lambda}, B=B_{\lambda}, C=C_{\lambda}, D=D_{\lambda}$ are to be determined. Thus, to solve problem (3.10) is equivalent to find $A, B, C, D$ to the following algebraic system:

$$
\left\{\begin{array}{l}
\left(-\rho_{1}+k \alpha^{2}\right) \lambda^{2} A-k \alpha \lambda B+\sigma \alpha \lambda C=0, \\
-k \alpha \lambda A+\left(\left(-\rho_{2}+b \alpha^{2}\right) \lambda^{2}+k\right) B-\sigma C=\rho_{2}, \\
-i \sigma \alpha \lambda A+i \sigma B+i \rho_{3} C-\alpha D=0 \\
\alpha \lambda C+(i \tau \lambda+\beta) D=0 .
\end{array}\right.
$$

We can rewrite this system as

$$
\underbrace{\left[\begin{array}{ccc}
p_{1}(\lambda) & -k \alpha \lambda & \sigma \alpha \lambda \\
-k \alpha \lambda & p_{2}(\lambda) & -\sigma \\
-i \sigma \alpha \lambda & i \sigma & p_{3}(\lambda)
\end{array}\right]}_{:=M}\left[\begin{array}{c}
A \\
B \\
C
\end{array}\right]=\left[\begin{array}{c}
0 \\
\rho_{2} \\
0
\end{array}\right] .
$$


where we set

$$
\left\{\begin{array}{l}
p_{1}(\lambda)=\left(-\rho_{1}+k \alpha^{2}\right) \lambda^{2} \\
p_{2}(\lambda)=\left(-\rho_{2}+b \alpha^{2}\right) \lambda^{2}+k, \\
p_{3}(\lambda)=i \rho_{3}+\frac{\alpha^{2} \lambda}{i \tau \lambda+\beta} .
\end{array}\right.
$$

A simple computation leads to

$$
\operatorname{det} M=\left[p_{1}(\lambda) p_{2}(\lambda)-k^{2} \alpha^{2} \lambda^{2}\right] p_{3}(\lambda)+i \sigma^{2} \alpha^{2} \lambda^{2}\left[p_{2}(\lambda)-2 k\right]+i \sigma^{2} p_{1}(\lambda),
$$

and

$$
B=\frac{\rho_{2}\left[p_{1}(\lambda) p_{3}(\lambda)+i \sigma^{2} \alpha^{2} \lambda^{2}\right]}{\operatorname{det} M} .
$$

Here we observe that $\operatorname{det} M \neq 0$, since

$$
\operatorname{Re} \operatorname{det} M=-\frac{\rho_{1} k \beta \alpha^{2} \lambda^{2}}{\beta^{2}+\tau^{2} \lambda^{2}} \neq 0 .
$$

Thus, noting that $p_{1}(\lambda)=k\left(\frac{\rho_{2}}{b}-\frac{\rho_{1}}{k}\right) \lambda^{2}, p_{2}(\lambda)=k$, we obtain

$$
B=\frac{\rho_{2} k\left(\frac{\rho_{2}}{b}-\frac{\rho_{1}}{k}\right) \lambda^{2} p_{3}(\lambda)+i \rho_{2} \sigma^{2} \alpha^{2} \lambda^{2}}{-\rho_{1} k \lambda^{2} p_{3}(\lambda)-i k \sigma^{2} \alpha^{2} \lambda^{2}+i k \sigma^{2}\left(\frac{\rho_{2}}{b}-\frac{\rho_{1}}{k}\right) \lambda^{2}},
$$

from where we obtain that $|B|=\left|B_{\lambda_{n}}\right|$ behaves like a constant as $n \rightarrow \infty$, no matter whether (1.2) holds or not, that is,

$$
|B| \approx c_{0}>0, \quad \text { as } n \rightarrow \infty .
$$

Having in mind that $\Psi(x)=i \lambda_{n} \psi(x)=i \lambda B_{\lambda_{n}} \sin \left(\alpha \lambda_{n} x\right)$, we obtain

$$
\left\|U_{n}\right\|_{\mathcal{H}_{C_{1}}}^{2} \geq \rho_{2} \int_{0}^{L}|\Psi(x)|^{2} d x=\rho_{2}\left|B_{\lambda_{n}}\right|^{2} \lambda_{n}^{2} \int_{0}^{L} \sin ^{2}\left(\alpha \lambda_{n} x\right) d x=\frac{\rho_{2} L}{2}\left|B_{\lambda_{n}}\right|^{2} \lambda_{n}^{2},
$$

which is enough to reach the desired limit (3.8).

Therefore, the proof of Theorem 3.1 is completed.

Remark 3.2. This kind of loss of exponential stability when going from Fourier's to Cattaneo's has been observed in [14] under damping on the bending moment, but also for thermoelastic plates in [23, 13]. In [24] it was outlined that this phenomenon might be more likely than not losing the exponential stability.

This loss of exponential stability was also, surprisingly, observed in [14] if, additionally, a history term is present, meaning an unexpected "destruction" by the Cattaneo heat conduction, as explained in the Introduction. Now, in the next Section 4 we will add the history term, but, interestingly, the system remains exponentially stable as in the Fourier case, in contrast to [14]. 


\section{Cattaneo with history: exponential stability}

In this section we study the case of Cattaneo's heat conduction law with a history term (1.17),

$$
\begin{cases}\rho_{1} \varphi_{t t}-k\left(\varphi_{x}+\psi\right)_{x}+\sigma \theta_{x}=0 & \text { in }(0, L) \times \mathbb{R}^{+}, \\ \rho_{2} \psi_{t t}-\tilde{b} \psi_{x x}+k\left(\varphi_{x}+\psi\right)-\int_{0}^{\infty} g(s) \eta_{x x}(s) d s-\sigma \theta=0 & \text { in }(0, L) \times \mathbb{R}^{+}, \\ \rho_{3} \theta_{t}+q_{x}+\sigma\left(\varphi_{x}+\psi\right)_{t}=0 & \text { in }(0, L) \times \mathbb{R}^{+}, \\ \tau q_{t}+\beta q+\theta_{x}=0 & \text { in }(0, L) \times \mathbb{R}^{+}, \\ \eta_{t}+\eta_{s}-\psi_{t}=0 & \text { in }(0, L) \times \mathbb{R}^{+} \times \mathbb{R}^{+}\end{cases}
$$

with initial-boundary conditions

$$
\left\{\begin{array}{l}
\varphi_{x}(0, t)=\varphi_{x}(L, t)=\psi(0, t)=\psi(L, t)=\theta(0, t)=\theta(L, t)=0 \\
\eta^{t}(0, s)=\eta^{t}(L, s)=0, \eta^{t}(\cdot, 0)=0, \quad t \geq 0, s>0
\end{array}\right.
$$

and

$$
\left\{\begin{array}{l}
\varphi(x, 0)=\varphi_{0}(x), \varphi_{t}(x, 0)=\varphi_{1}(x), \psi(x, 0)=\psi_{0}(x), \psi_{t}(x, 0)=\psi_{1}(x), \\
\theta(x, 0)=\theta_{0}(x), q(x, 0)=q_{0}(x), \eta^{0}(x, s)=\eta_{0}(x, s), \quad x \in(0, L), s>0 .
\end{array}\right.
$$

The assumption on the kernel $g$ will be again Assumption 2.1, as in Section 2 .

To address problem (4.1)-(4.3), we consider the phase (Hilbert) space

$$
\mathcal{H}_{C_{2}}:=H_{*}^{1} \times L_{*}^{2} \times H_{0}^{1} \times L^{2} \times L^{2} \times L^{2} \times L_{g}^{2}\left(\mathbb{R}^{+}, H_{0}^{1}\right),
$$

equipped with the norm

$$
\|U\|_{\mathcal{H}_{C_{2}}}^{2} \equiv \rho_{1}\|\Phi\|_{2}^{2}+\rho_{2}\|\Psi\|_{2}^{2}+k\left\|\varphi_{x}+\psi\right\|_{2}^{2}+\tilde{b}\left\|\psi_{x}\right\|_{2}^{2}+\rho_{3}\|\theta\|_{2}^{2}+\tau\|q\|_{2}^{2}+\|\eta\|_{L_{g}^{2}}^{2},
$$

for $U:=(\varphi, \Phi, \psi, \Psi, \theta, q, \eta) \in \mathcal{H}_{C_{2}}$. Thus, denoting $\Phi=\varphi_{t}$ and $\Psi=\psi_{t}$, we can transform problem (4.1)-(4.3) into the first-order system

$$
\left\{\begin{array}{l}
U_{t}=\mathcal{A}_{C_{2}} U, \quad t>0 \\
U(0)=\left(\varphi_{0}, \varphi_{1}, \psi_{0}, \psi_{1}, \theta_{0}, q_{0}, \eta_{0}\right)=: U_{0}
\end{array}\right.
$$

where $\mathcal{A}_{C_{2}}: D\left(\mathcal{A}_{C_{2}}\right) \subset \mathcal{H}_{C_{2}} \rightarrow \mathcal{H}_{C_{2}}$ is given by

$$
\mathcal{A}_{C_{2}} U:=\left[\begin{array}{c}
\Phi \\
\frac{k}{\rho_{1}}\left(\varphi_{x}+\psi\right)_{x}-\frac{\sigma}{\rho_{1}} \theta_{x} \\
\Psi \\
\frac{1}{\rho_{2}}\left(\tilde{b} \psi+\int_{0}^{\infty} g(s) \eta(s) d s\right)_{x x}-\frac{k}{\rho_{2}}\left(\varphi_{x}+\psi\right)+\frac{\sigma}{\rho_{2}} \theta \\
-\frac{1}{\rho_{3}} q_{x}-\frac{\sigma}{\rho_{3}}\left(\Phi_{x}+\Psi\right) \\
-\frac{\beta}{\tau} q-\frac{1}{\tau} \theta_{x} \\
\Psi-\eta_{s}
\end{array}\right]
$$


with domain

$$
\begin{aligned}
D\left(\mathcal{A}_{C_{2}}\right):=\left\{U \in \mathcal{H}_{C_{2}} \mid \Phi\right. & \in H_{*}^{1}, \varphi_{x}, \Psi, \theta \in H_{0}^{1}, \eta_{s} \in L_{g}^{2}\left(\mathbb{R}^{+}, H_{0}^{1}\right), \\
q & \left.\in H^{1}, \varphi, \tilde{b} \psi+\int_{0}^{\infty} g(s) \eta(s) d s \in H^{2}, \eta(\cdot, 0)=0\right\} .
\end{aligned}
$$

It is not difficult to prove that $0 \in \varrho\left(\mathcal{A}_{C_{2}}\right)$ and $\mathcal{A}_{C_{2}}$ is dissipative, with

$$
\begin{aligned}
\operatorname{Re}\left(\mathcal{A}_{C_{2}} U, U\right)_{\mathcal{H}_{C_{2}}} & =-\beta\|q\|_{2}^{2}+\frac{1}{2} \int_{0}^{\infty} g^{\prime}(s)\left\|\eta_{x}(s)\right\|_{2}^{2} d s \\
& \leq-\beta\|q\|_{2}^{2}-\frac{k_{1}}{2}\|\eta\|_{L_{g}^{2}}^{2} \\
& \leq 0, \quad \forall U \in D\left(\mathcal{A}_{C_{2}}\right),
\end{aligned}
$$

and we have again a unique solution to to problem (4.5) as in Section $2, U(t) \equiv e^{\mathcal{A}_{C_{2}}{ }^{t} U_{0}}$. We shall prove that the semigroup $\left\{e^{\mathcal{A}_{C_{2}}{ }^{t}}\right\}_{t \geq 0}$ is exponentially stable.

Theorem 4.1. Under the Assumption 2.1, there exist constants $C, \gamma>0$ independent of $U_{0} \in$ $\mathcal{H}_{C_{2}}$ such that for all $t \geq 0$

$$
\|U(t)\|_{\mathcal{H}_{C_{2}}} \leq C\left\|U_{0}\right\|_{\mathcal{H}_{C_{2}}} e^{-\gamma t} .
$$

In other words, the thermo-viscoelastic Timoshenko system under the Cattaneo law (4.1)-(4.3) is exponentially stable independent of any relation between the coefficients.

To the proof of Theorem 4.1, we still use Theorem 2.3. It will follow as a consequence of the following steps.

\subsection{Verifying (2.13): The resolvent set $\varrho\left(\mathcal{A}_{F}\right)$ contains the imaginary axis}

In order to prove that

$$
i \mathbb{R} \subset \varrho\left(\mathcal{A}_{C_{2}}\right),
$$

let us argue again by contradiction argument. We assume that $i \mathbb{R} \not \subset \varrho\left(\mathcal{A}_{C_{2}}\right)$ and conclude the existence of a constant $\omega>0$, w.l.o.g., and a sequence $\lambda_{n} \in \mathbb{R}$, with $0<\lambda_{n} \rightarrow w$ from below and $i \lambda_{n} \in \varrho\left(\mathcal{A}_{F}\right)$, and a sequence of functions

$$
U_{n}=\left(\varphi_{n}, \Phi_{n}, \psi_{n}, \Psi_{n}, \theta_{n}, q_{n}, \eta_{n}\right) \in D\left(\mathcal{A}_{C_{2}}\right) \quad \text { with } \quad\left\|U_{n}\right\|_{\mathcal{H}_{C_{2}}}=1,
$$

such that

$$
i \lambda_{n} U_{n}-\mathcal{A}_{C_{2}} U_{n} \rightarrow 0 \text { in } \mathcal{H}_{C_{2}} .
$$

In view of $\mathcal{A}_{C_{2}}$ defined in (4.6), the limit (4.11) yields

$$
\begin{cases}i \lambda_{n} \varphi_{n}-\Phi_{n} \rightarrow 0 & \text { in } H_{*}^{1}(0, L), \\ i \lambda_{n} \rho_{1} \Phi_{n}-k\left(\varphi_{n, x}+\psi_{n}\right)_{x}+\sigma \theta_{n, x} \rightarrow 0 & \text { in } L_{*}^{2}(0, L), \\ i \lambda_{n} \psi_{n}-\Psi_{n} \rightarrow 0 & \text { in } H_{0}^{1}(0, L), \\ i \lambda_{n} \rho_{2} \Psi_{n}+k\left(\varphi_{n, x}+\psi_{n}\right) & \\ \quad-\left(\tilde{b} \psi_{n}+\int_{0}^{\infty} g(s) \eta_{n}(s) d s\right)_{x x}-\sigma \theta_{n} \rightarrow 0 & \text { in } \quad L^{2}(0, L), \\ i \lambda_{n} \rho_{3} \theta_{n}+q_{n, x}+\sigma\left(\Phi_{n, x}+\Psi_{n}\right) \rightarrow 0 & \text { in } L^{2}(0, L), \\ i \lambda_{n} \tau q_{n}+\beta q_{n}+\theta_{n, x} \rightarrow 0 & \text { in } \quad L^{2}(0, L), \\ i \lambda_{n} \eta_{n}+\eta_{n, s}-\Psi_{n} \rightarrow 0 & \text { in } \quad L_{g}^{2}\left(\mathbb{R}^{+}, H_{0}^{1}(0, L)\right) .\end{cases}
$$


Lemma 4.2. Under the assumptions of Theorem 4.1 we have, as $n \rightarrow \infty$ :

$$
\begin{aligned}
& \left\|q_{n}\right\|_{2} \rightarrow 0 \\
& \int_{0}^{\infty}\left[-g^{\prime}(s)\right]\left\|\eta_{n, x}(s)\right\|_{2}^{2} d s \rightarrow 0, \\
& \left\|\eta_{n}\right\|_{L_{g}^{2}} \rightarrow 0 \\
& \left\|\theta_{n, x}\right\|_{2} \rightarrow 0
\end{aligned}
$$

Proof. The limits (4.13)-(4.15) arise directly from (4.7) and (4.11). Additionally, (4.16) is a consequence of (4.13) and (4.12).

From the limits the of Lemma 4.2, (4.12) can be reduced to the following

$$
\begin{aligned}
i \lambda_{n} \varphi_{n}-\Phi_{n} \rightarrow 0 & \text { in } \quad H_{*}^{1}(0, L), \\
i \lambda_{n} \rho_{1} \Phi_{n}-k\left(\varphi_{n, x}+\psi_{n}\right)_{x} \rightarrow 0 & \text { in } L_{*}^{2}(0, L), \\
i \lambda_{n} \psi_{n}-\Psi_{n} \rightarrow 0 & \text { in } H_{0}^{1}(0, L), \\
i \lambda_{n} \rho_{2} \Psi_{n}+k\left(\varphi_{n, x}+\psi_{n}\right)-\left(\tilde{b} \psi_{n}+\int_{0}^{\infty} g(s) \eta_{n}(s) d s\right)_{x x} \rightarrow 0 & \text { in } L^{2}(0, L), \\
i \lambda_{n} \eta_{n}+\eta_{n, s}-\Psi_{n} \rightarrow 0 & \text { in } \quad L_{g}^{2}\left(\mathbb{R}^{+}, H_{0}^{1}(0, L)\right),
\end{aligned}
$$

which are precisely the same limits as given in (2.22a)-(2.22e). Therefore, in what follows, the arguments are the same as presented previously.

Lemma 4.3. Under the assumptions of Theorem 4.1 we have:

$$
\left\|\Psi_{n, x}\right\|_{2}^{2},\left\|\psi_{n, x}\right\|_{2}^{2},\left\|\varphi_{n, x}+\psi_{n}\right\|_{2}^{2},\left\|\Phi_{n}\right\|_{2}^{2} \rightarrow 0, \text { as } n \rightarrow \infty
$$

Proof. It follows verbatim with the same arguments as in the proofs of Lemmas 2.5 and 2.6.

Therefore, combining (4.13), (4.15), (4.16) and (4.17), we conclude that

$$
\left\|U_{n}\right\|_{\mathcal{H}_{C_{2}}} \rightarrow 0
$$

yielding the desired contradiction with (4.10). This finishes the proof of (4.9).

\subsection{Verifying (2.14): Boundedness of $\left(i \lambda I_{d}-\mathcal{A}_{F}\right)^{-1}$}

Let us prove that there exists a constant $C>0$, independent of $\lambda$, such that, as $|\lambda| \rightarrow \infty$,

$$
\left\|\left(i \lambda I_{d}-\mathcal{A}_{C_{2}}\right)^{-1}\right\|_{\mathcal{L}\left(\mathcal{H}_{C_{2}}\right)} \leq C .
$$

To this end, let $\Upsilon=\left(f_{1}, \ldots, f_{7}\right) \in \mathcal{H}_{C_{2}}$ be given, and let $U \in D\left(\mathcal{A}_{C_{2}}\right)$ be the solution of

$$
i \lambda U-\mathcal{A}_{C_{2}} U=\Upsilon
$$


which in terms of its components reads

$$
\begin{aligned}
& i \lambda \varphi-\Phi=f_{1}, \\
& i \lambda \rho_{1} \Phi-k\left(\varphi_{x}+\psi\right)_{x}+\sigma \theta_{x}=\rho_{1} f_{2}, \\
& i \lambda \psi-\Psi=f_{3}, \\
& i \lambda \rho_{2} \Psi-\tilde{b} \psi_{x x}-\int_{0}^{\infty} g(s) \eta_{x x}(s) d s+k\left(\varphi_{x}+\psi\right)-\sigma \theta=\rho_{2} f_{4}, \\
& i \lambda \rho_{3} \theta+q_{x}+\sigma\left(\Phi_{x}+\Psi\right)=\rho_{3} f_{5}, \\
& i \lambda \tau q+\beta q+\theta_{x}=\tau f_{6}, \\
& i \lambda \eta+\eta_{s}-\Psi=f_{7} .
\end{aligned}
$$

We have to show that there exists a constant $C>0$, independent of $\lambda$, such that, as $|\lambda| \rightarrow \infty$,

$$
\|U\|_{\mathcal{H}_{C_{2}}} \leq C\|\Upsilon\|_{\mathcal{H}_{C_{2}}}
$$

Lemma 4.4. Under the assumptions of Theorem 4.1, there exists a constant $C>0$, independent of $\lambda$, such that

$$
\|q\|_{2}^{2}, \int_{0}^{\infty}\left[-g^{\prime}(s)\right]\left\|\eta_{x}(s)\right\|_{2}^{2} d s \leq C\|U\|_{\mathcal{H}_{C_{2}}}\|\Upsilon\|_{\mathcal{H}_{C_{2}}}
$$

In particular, there exists a constant $C>0$, independent of $\lambda$, such that

$$
\|\eta\|_{L_{g}^{2}}^{2} \leq C\|U\|_{\mathcal{H}_{C_{2}}}\|\Upsilon\|_{\mathcal{H}_{C_{2}}}
$$

Proof. As before in Section 2, this follows promptly from the dissipativity (4.7).

Lemma 4.5. Under the assumptions of Theorem 4.1, there exists a constant $C>0$, independent of $\lambda$, such that

$$
\rho_{3}\|\theta\|_{2}^{2} \leq C\|U\|_{\mathcal{H}_{C_{2}}}\|\Upsilon\|_{\mathcal{H}_{C_{2}}}+C\|q\|_{2}\|\Phi\|_{2}+C\|q\|_{2}\|\Psi\|_{2} .
$$

In particular, given any $\epsilon>0$, there exists a constant $C_{\epsilon}>0$, independent of $\lambda$, such that

$$
\rho_{3}\|\theta\|_{2}^{2} \leq \epsilon\|U\|_{\mathcal{H}_{C_{2}}}^{2}+C_{\epsilon}\|\Upsilon\|_{\mathcal{H}_{C_{2}}}^{2} .
$$

Proof. Integrating (4.20f) over $(0, x) \subset(0, L)$ and taking the multiplier $\bar{\theta}$ in the resulting expression, we get

$$
\begin{aligned}
\int_{0}^{L}|\theta|^{2} d x= & \underbrace{\tau \int_{0}^{L} \int_{0}^{x} q(y) d y \overline{(i \lambda \theta(x))} d x}_{=: S_{1}}-\beta \int_{0}^{L} \int_{0}^{x} q(y) d y \overline{\theta(x)} d x+ \\
& \tau \int_{0}^{L} \int_{0}^{x} f_{6}(y) d y \overline{\theta(x)} d x .
\end{aligned}
$$

From (4.20e) we can rewrite $S_{1}$ as follows

$$
\begin{aligned}
S_{1}= & \frac{\tau}{\rho_{3}} \int_{0}^{L}|q|^{2} d x-\frac{\tau}{\rho_{3}}(\bar{q}(L)+\sigma \bar{\Phi}(L)) \int_{0}^{L} q d x \\
& +\frac{\tau \sigma}{\rho_{3}} \int_{0}^{L} q \bar{\Phi} d x-\frac{\tau \sigma}{\rho_{3}} \int_{0}^{L} \int_{0}^{x} q(y) d y \bar{\Psi}(x) d x+\tau \int_{0}^{L} \int_{0}^{x} q(y) d y \overline{f_{5}}(x) d x .
\end{aligned}
$$


Replacing this in (4.26) we obtain

$$
\begin{aligned}
\rho_{3} \int_{0}^{L}|\theta|^{2} d x= & \tau \int_{0}^{L}|q|^{2} d x-\rho_{3} \beta \int_{0}^{L} \int_{0}^{x} q(y) d y \bar{\theta}(x) d x-\tau \overbrace{(\bar{q}(L)+\sigma \bar{\Phi}(L)) \int_{0}^{L} q d x}^{=: S_{2}} \\
& +\tau \sigma \int_{0}^{L} q \bar{\Phi} d x-\tau \sigma \int_{0}^{L} \int_{0}^{x} q(y) d y \bar{\Psi}(x) d x \\
& +\rho_{3} \tau \int_{0}^{L} \int_{0}^{x} q(y) d y \overline{f_{5}}(x) d x+\rho_{3} \tau \int_{0}^{L} \int_{0}^{x} f_{6}(y) d y \bar{\theta}(x) d x .
\end{aligned}
$$

In what follows we are going to estimate $S_{2}$. Indeed, integrating $(4.20 \mathrm{e})$ on $(x, L)$, taking the multiplier $\int_{0}^{L} \bar{q} d x$ in the resulting expression and then rearranging the terms, we obtain

$$
\begin{aligned}
{[q(L)+\sigma \Psi(L)] \int_{0}^{L} \bar{q} d x=} & \rho_{3} \int_{x}^{L} f_{5}(s) d s \int_{0}^{L} \bar{q}(z) d z+[q(x)+\sigma \Phi(x)] \int_{0}^{L} \bar{q}(z) d z \\
& +\underbrace{\rho_{3} \int_{x}^{L} \theta(s) d s \int_{0}^{L} \overline{(i \lambda q)}(z) d z}_{=: S_{3}}-\sigma \int_{x}^{L} \Psi(s) d s \int_{0}^{L} \bar{q}(z) d z .
\end{aligned}
$$

Now, using the identity (4.20f) in $S_{3}$ and noting that $\theta \in H_{0}^{1}(0, L)$, we can rewrite (4.28) as follows.

$$
\begin{aligned}
\overbrace{[q(L)+\sigma \Psi(L)] \int_{0}^{L} \bar{q}(z) d z}^{=\overline{S_{2}}}= & \rho_{3} \int_{x}^{L} f_{5}(s) d s \int_{0}^{L} \bar{q}(z) d z+[q(x)+\sigma \Phi(x)] \int_{0}^{L} \bar{q}(z) d z \\
& +\frac{\rho_{3}}{\tau} \int_{x}^{L} \theta(s) d s \int_{0}^{L}\left(\tau \overline{f_{6}}-\beta \bar{q}\right)(z) d z-\sigma \int_{x}^{L} \Psi(s) d s \int_{0}^{L} \bar{q}(z) d z,
\end{aligned}
$$

and integrating this expression with respect to $x$ on $(0, L)$, we easily deduce by $(4.22)$

$$
\left|S_{2}\right| \leq C\|U\|_{\mathcal{H}_{C_{2}}}\|\Upsilon\|_{\mathcal{H}_{C_{2}}}+C\|q\|_{2}\|\Phi\|_{2}+C\|q\|_{2}\|\Psi\|_{2}+C\|q\|_{2}\|\theta\|_{2},
$$

for some constant $C>0$. Thus, going back to (4.27) we arrive at

$$
\rho_{3}\|\theta\|_{2}^{2} \leq C\|U\|_{\mathcal{H}_{C_{2}}}\|\Upsilon\|_{\mathcal{H}_{C_{2}}}+C\|q\|_{2}\|\Phi\|_{2}+C\|q\|_{2}\|\Psi\|_{2}+C\|q\|_{2}\|\theta\|_{2},
$$

for some constant $C>0$. Therefore, using (4.22), we conclude that (4.24) holds true and, consequently, (4.25).

Lemma 4.6. Under the assumptions of Theorem 4.1 and given any $\epsilon>0$, there exists a constant $C_{\epsilon}>0$, independent of $\lambda$, such that

$$
k\left\|\varphi_{x}+\psi\right\|_{2}^{2} \leq \epsilon\|U\|_{\mathcal{H}_{C_{2}}}^{2}+C_{\epsilon}\|\Upsilon\|_{\mathcal{H}_{C_{2}}}^{2}
$$

for $|\lambda|>1$.

Proof. Substituting the resolvent equations (4.20a) and (4.20c) in (4.20e), we have

$$
i \lambda \rho_{3} \theta+q_{x}+i \lambda \sigma\left(\varphi_{x}+\psi\right)=\rho_{3} f_{5}+\sigma\left(f_{1, x}+f_{3}\right) .
$$


Multiplying (4.31) by $k \overline{\left(\varphi_{x}+\psi\right)}$ and integrating over $(0, L)$, we get

$$
\begin{aligned}
& i \lambda \sigma k \int_{0}^{L}\left|\varphi_{x}+\psi\right|^{2} d x=\underbrace{\int_{0}^{L} q \overline{\left[k\left(\varphi_{x}+\psi\right)_{x}\right]} d x}_{=: S_{4}}-i \lambda \rho_{3} k \int_{0}^{L} \theta \overline{\left(\varphi_{x}+\psi\right)} d x \\
& +k \int_{0}^{L}\left[\rho_{3} f_{5}+\sigma\left(f_{1, x}+f_{3}\right)\right] \overline{\left(\varphi_{x}+\psi\right)} d x .
\end{aligned}
$$

Let us rewrite the terms $S_{4}$ as follows. Using (4.20b) and then (4.20f), we infer

$$
\begin{aligned}
S_{4}= & -i \lambda \rho_{1} \int_{0}^{L} q \bar{\Phi} d x+\sigma \int_{0}^{L} q \overline{\theta_{x}} d x-\rho_{1} \int_{0}^{L} q \overline{f_{2}} d x \\
= & -i \lambda \rho_{1} \int_{0}^{L} q \bar{\Phi} d x+i \lambda \tau \sigma \int_{0}^{L}|q|^{2} d x-\beta \sigma \int_{0}^{L}|q|^{2} d x \\
& +\tau \sigma \int_{0}^{L} q \overline{f_{6}} d x-\rho_{1} \int_{0}^{L} q \overline{f_{2}} d x .
\end{aligned}
$$

Replacing this in (4.32) it follows that

$$
\begin{aligned}
i \lambda \sigma k\left\|\varphi_{x}+\psi\right\|_{2}^{2}= & -i \lambda \rho_{1} \int_{0}^{L} q \bar{\Phi} d x-i \lambda \rho_{3} k \int_{0}^{L} \theta \overline{\left(\varphi_{x}+\psi\right)} d x \\
& +(i \lambda \tau-\beta) \sigma\|q\|_{2}^{2}+S_{5}
\end{aligned}
$$

where we denote

$$
S_{5}:=\tau \sigma \int_{0}^{L} q \overline{f_{6}} d x-\rho_{1} \int_{0}^{L} q \overline{f_{2}} d x+k \int_{0}^{L}\left[\rho_{3} f_{5}+\sigma\left(f_{1, x}+f_{3}\right)\right] \overline{\left(\varphi_{x}+\psi\right)} d x .
$$

We have

$$
\left|S_{5}\right| \leq C\|U\|_{\mathcal{H}_{C_{2}}}\|\Upsilon\|_{\mathcal{H}_{C_{2}}}
$$

Now, from the identity (4.33), estimates (4.22) and (4.24), Young's inequality and $|\lambda|>1$, we obtain

$$
k\left\|\varphi_{x}+\psi\right\|_{2}^{2} \leq C\|q\|_{2}\|\Phi\|_{2}+C\|q\|_{2}\|\Psi\|_{2}+C\|\theta\|_{2}\|U\|_{\mathcal{H}_{C_{2}}}+C\|U\|_{\mathcal{H}_{C_{2}}}\|\Upsilon\|_{\mathcal{H}_{C_{2}}}
$$

for some constant $C>0$. Finally, from (4.34) and combining again (4.22), (4.24) and Young's inequality with $\epsilon>0$, we conclude (4.30) for $|\lambda|>1$.

Lemma 4.7. Under the assumptions of Theorem 4.1 and given any $\epsilon>0$, there exists a constant $C_{\epsilon}>0$, independent of $\lambda$, such that

$$
\rho_{1}\|\Phi\|_{2}^{2} \leq \epsilon\|U\|_{\mathcal{H}_{C_{2}}}^{2}+C_{\epsilon}\|\Upsilon\|_{\mathcal{H}_{C_{2}}}^{2}
$$

for $|\lambda|>1$ large enough.

Proof. Multiplying (4.20b) by $-\bar{\varphi}$, integrating on $(0, L)$, using (4.20a), adding and subtracting $k \int_{0}^{L}\left(\varphi_{x}+\psi\right) \bar{\psi} d x$, we get

$$
\begin{aligned}
\rho_{1} \int_{0}^{L}|\Phi|^{2} d x= & k \int_{0}^{L}\left|\varphi_{x}+\psi\right|^{2} d x-k \int_{0}^{L}\left(\varphi_{x}+\psi\right) \bar{\psi} d x \\
& +\underbrace{\sigma \int_{0}^{L}\left[\frac{i}{\lambda} \theta_{x}\right] \overline{\left(\Phi+f_{1}\right)} d x}_{:=S_{6}}-\rho_{1} \int_{0}^{L}\left(\Phi \overline{f_{1}}+f_{2} \bar{\varphi}\right) d x
\end{aligned}
$$


Using (4.20f) for $S_{6}$, one has

$$
\begin{aligned}
\rho_{1} \int_{0}^{L}|\Phi|^{2} d x= & k \int_{0}^{L}\left|\varphi_{x}+\psi\right|^{2} d x-k \int_{0}^{L}\left(\varphi_{x}+\psi\right) \bar{\psi} d x \\
& +\sigma\left(\tau-\frac{i \beta}{\lambda}\right) \int_{0}^{L} q \bar{\Phi} d x+S_{7},
\end{aligned}
$$

where

$$
S_{7}:=\frac{i \sigma \tau}{\lambda} \int_{0}^{L} f_{6} \overline{\left(\Phi+f_{1}\right)} d x+\sigma\left(\tau-\frac{i \beta}{\lambda}\right) \int_{0}^{L} q \overline{f_{1}} d x-\rho_{1} \int_{0}^{L}\left(\Phi \overline{f_{1}}+f_{2} \bar{\varphi}\right) d x .
$$

We have

$$
\left|S_{5}\right| \leq C\|U\|_{\mathcal{H}_{C_{2}}}\|\Upsilon\|_{\mathcal{H}_{C_{2}}}+C\|\Upsilon\|_{\mathcal{H}_{C_{2}}}^{2},
$$

for some constant $C>0$ and $|\lambda|>1$. Returning to (4.37), using (4.20c) and (4.34), one gets

$$
\begin{aligned}
\rho_{1}\|\Phi\|_{2}^{2} \leq & C\|q\|_{2}\|U\|_{\mathcal{H}_{C_{2}}}+C\|\theta\|_{2}\|U\|_{\mathcal{H}_{C_{2}}} \\
& +C\|U\|_{\mathcal{H}_{C_{2}}}\|\Upsilon\|_{\mathcal{H}_{C_{2}}}+\frac{C}{|\lambda|^{2}}\|U\|_{\mathcal{H}_{C_{2}}}^{2}+C\|\Upsilon\|_{\mathcal{H}_{C_{2}}}^{2},
\end{aligned}
$$

for $|\lambda|>1$ and some constant $C>0$. Therefore, the conclusion of (4.35) follows analogously as in the previous lemmas and taking $|\lambda|>1$ sufficiently large.

Lemma 4.8. Under the assumptions of Theorem 4.1 and given any $\epsilon>0$, there exists a constant $C_{\epsilon}>0$, independent of $\lambda$, such that

$$
\rho_{2}\|\Psi\|_{2}^{2} \leq \epsilon\|U\|_{\mathcal{H}_{C_{2}}}^{2}+C_{\epsilon}\|\Upsilon\|_{\mathcal{H}_{C_{2}}}^{2}
$$

for $|\lambda|>1$ large enough.

Proof. Multiplying (4.20d) by $\int_{0}^{\infty} g(s) \overline{\eta(s)} d s$ and integrating on $(0, L)$, we get

$$
\begin{gathered}
\underbrace{-\rho_{2} \int_{0}^{L} \int_{0}^{\infty} g(s) \Psi \overline{[i \lambda \eta(s)]} d s d x}_{=: S 8}-\sigma \int_{0}^{L} \int_{0}^{\infty} g(s) \theta \overline{\eta(s)} d s d x+\tilde{b} \int_{0}^{L} \int_{0}^{\infty} g(s) \overline{\eta_{x}(s)} \psi_{x} d s d x \\
+\int_{0}^{L}\left|\int_{0}^{\infty} g(s) \eta_{x}(s) d s\right|^{2} d x+\underbrace{k \int_{0}^{L} \int_{0}^{\infty} g(s)\left(\varphi_{x}+\psi\right) \overline{\eta(s)} d s d x}_{=: S_{9}}=\rho_{2} \int_{0}^{L} \int_{0}^{\infty} g(s) f_{4} \overline{\eta(s)} d s d x .
\end{gathered}
$$

Now, using the identity (4.20g) in $S_{8}$ and the expressions (4.20a) and (4.20c) in $S_{9}$, we obtain

$$
\begin{aligned}
-\overbrace{2} \overbrace{\left(\int_{0}^{\infty} g(s) d s\right)}^{=b_{0}} \int_{0}^{L}|\Psi|^{2} d x= & \sigma \int_{0}^{L} \int_{0}^{\infty} g(s) \theta \overline{\eta(s)} d s d x-\tilde{b} \int_{0}^{L} \int_{0}^{\infty} g(s) \overline{\eta_{x}(s)} \psi_{x} d s d x \\
& +\rho_{2} \int_{0}^{L} \int_{0}^{\infty} g(s) f_{4} \overline{\eta(s)} d s d x+\rho_{2} \int_{0}^{L} \int_{0}^{\infty} g(s) \Psi \overline{f_{7}} d s d x \\
& +S_{10}+S_{11}+S_{12}
\end{aligned}
$$


where we denote

$$
S_{10}:=-\rho_{2} \int_{0}^{L} \int_{0}^{\infty} g(s) \Psi \overline{\left[\eta_{s}(s)\right]} d s d x, \quad S_{11}:=-\int_{0}^{L}\left(\int_{0}^{\infty} g(s) \eta_{x}(s) d s\right)^{2} d x,
$$

and

$$
\begin{aligned}
S_{12}:= & +\frac{i k}{\lambda} \int_{0}^{L} \int_{0}^{\infty} g(s) \overline{\eta_{x}(s)} d s \Phi d x-\frac{i k}{\lambda} \int_{0}^{L} \int_{0}^{\infty} g(s) \overline{\eta(s)} d s \Psi d x \\
& +\frac{i k}{\lambda} \int_{0}^{L} \int_{0}^{\infty} g(s) \overline{\eta(s)} d s\left(f_{1, x}+f_{3}\right) d x .
\end{aligned}
$$

Let us estimate $S_{10}, S_{11}$ and $S_{12}$ as follows.

$$
\begin{gathered}
\left|S_{10}\right| \leq \rho_{2} b_{0}{ }^{1 / 2}\|\Psi\|_{2}\left(\int_{0}^{\infty}\left[-g^{\prime}(s)\right]\|\eta(s)\|_{2}^{2} d s\right)^{1 / 2}, \\
\left|S_{11}\right| \leq b_{0}\|\eta\|_{L_{g}^{2}}^{2}, \\
\left|S_{12}\right| \leq \frac{C}{|\lambda|}\|\eta\|_{L_{g}^{2}}\|\Phi\|_{2}+\frac{C}{|\lambda|}\|\eta\|_{L_{g}^{2}}\|\Psi\|_{2}+\frac{C}{|\lambda|}\|\eta\|_{L_{g}^{2}}\left\|f_{1, x}+f_{3}\right\|_{2} .
\end{gathered}
$$

Uing these last three estimates in (4.39) and using (4.23), we arrive at

$$
\begin{aligned}
\rho_{2}\|\Psi\|_{2}^{2} \leq & C\|\eta\|_{L_{g}^{2}}\|\theta\|_{2}+C\|\eta\|_{L_{g}^{2}}\left\|\psi_{x}\right\|_{2}+C\|U\|_{\mathcal{H}_{C_{2}}}\|\Upsilon\|_{\mathcal{H}_{C_{2}}} \\
& +C\|\Psi\|_{2}\left(\int_{0}^{\infty}\left[-g^{\prime}(s)\right]\left\|\eta_{x}(s)\right\|_{2}^{2} d s\right)^{1 / 2} \\
& +\frac{C}{|\lambda|}\|\eta\|_{L_{g}^{2}}\|\Phi\|_{2}+\frac{C}{|\lambda|}\|\eta\|_{L_{g}^{2}}\|\Psi\|_{2},
\end{aligned}
$$

for come constant $C>0$ and $|\lambda|>1$. Using Young's inequality once more and the estimates (4.22)-(4.23) we finally obtain

$$
\rho_{2}\|\Psi\|_{2}^{2} \leq C\|U\|_{\mathcal{H}_{C_{2}}}\|\Upsilon\|_{\mathcal{H}_{C_{2}}}+C\|\eta\|_{L_{g}^{2}}\|U\|_{\mathcal{H}_{C_{2}}},
$$

for come constant $C>0$ and $|\lambda|>1$. Finally, from (4.41), using again the estimate (4.23) and Young's inequality with $\epsilon>0$, we conclude (4.38).

Lemma 4.9. Under the assumptions of Theorem 4.1 and given any $\epsilon>0$, there exists a constant $C_{\epsilon}>0$, independent of $\lambda$, such that

$$
\tilde{b}\left\|\psi_{x}\right\|_{2}^{2} \leq \epsilon\|U\|_{\mathcal{H}_{C_{2}}}^{2}+C_{\epsilon}\|\Upsilon\|_{\mathcal{H}_{C_{2}}}^{2},
$$

for $|\lambda|>1$ large enough.

Proof. Multiplying (4.20d) by $\bar{\psi}$ and integrating, we get

$$
\begin{gathered}
\underbrace{-\rho_{2} \int_{0}^{L} \Psi \overline{(i \lambda \psi)} d x}_{=: S_{13}}+\tilde{b} \int_{0}^{L}\left|\psi_{x}\right|^{2} d x+\int_{0}^{L} \int_{0}^{\infty} g(s) \eta_{x}(s) \overline{\psi_{x}} d s d x \\
+\underbrace{k \int_{0}^{L}\left(\varphi_{x}+\psi\right) \bar{\psi} d x}_{=: S_{14}}-\sigma \int_{0}^{L} \theta \bar{\psi} d x=\rho_{2} \int_{0}^{L} f_{4} \bar{\psi} d x .
\end{gathered}
$$


Replacing $\psi$, given in the component equation (4.20c), in both $S_{13}$ and $S_{14}$, we have

$$
\begin{aligned}
\tilde{b} \int_{0}^{L}\left|\psi_{x}\right|^{2} d x= & -\int_{0}^{L} \int_{0}^{\infty} g(s) \eta_{x}(s) \overline{\psi_{x}} d s d x+\frac{i k}{\lambda} \int_{0}^{L}\left(\varphi_{x}+\psi\right) \bar{\Psi} d x \\
& +\sigma \int_{0}^{L} \theta \bar{\psi} d x+\rho_{2} \int_{0}^{L}|\Psi|^{2} d x+S_{15}
\end{aligned}
$$

where

$$
S_{15}:=\frac{i k}{\lambda} \int_{0}^{L}\left(\varphi_{x}+\psi\right) \overline{f_{3}} d x+\rho_{2} \int_{0}^{L} f_{4} \bar{\psi} d x+\rho_{2} \int_{0}^{L} \Psi \overline{f_{3}} d x
$$

hence

$$
\left|S_{15}\right| \leq C\|U\|_{\mathcal{H}_{C_{2}}}\|\Upsilon\|_{\mathcal{H}_{C_{2}}}
$$

for some constant $C>0$ and $|\lambda|>1$. From the latter and in combination with (4.34) and (4.41), we obtain from (4.43) the estimate

$$
\tilde{b}\left\|\psi_{x}\right\|_{2}^{2} \leq C\|\eta\|_{L_{g}^{2}}\|U\|_{\mathcal{H}_{C_{2}}}+C\|q\|_{2}\|U\|_{\mathcal{H}_{C_{2}}}+C\|\theta\|_{2}\|U\|_{\mathcal{H}_{C_{2}}}+C\|U\|_{\mathcal{H}_{C_{2}}}\|\Upsilon\|_{\mathcal{H}_{C_{2}}},
$$

for come constant $C>0$ and $|\lambda|>1$. Finally, applying Young's inequality several times and observing the useful estimates (4.22), (4.23) and (4.24), we conclude the estimate (4.42).

Last, combining the Lemmas 4.4-4.9 and choosing $\epsilon>0$ small enough, one can easily conclude (4.21).

This finishes the proof of Theorem 4.1.

Remark 4.10. Let us finally stress some technical aspects concerning the result provided by Theorem 4.1 when compared to [14] (see Sections 3 and 4 therein) and [11, Section 3]. Here, unlike [14, 11], our main result in this section features the exponential stability for (4.1)-(4.3) independent of any relation between the coefficients. The main technical reason for this achievement is that we can estimate the shear component (see Lemma 4.6) by means of the shear force damping, which leads to a new way of getting a priori estimates in comparison to bending moment damping, see e.g. [14, Lemma 4.4] and [11, Lemma 3.5]. Moreover, the same result (Theorem 4.1) can probably be extended to other boundary conditions instead of (4.2) so that the existence of solution is ensured in proper spaces. Although additional computations are necessary to this purpose, they can be done by following similar ideas as given in [21, 25] to control possible point-wise boundary terms or else we can follow the same lines as [4, 5] by introducing cut-off functions and get local estimates that could be globally expanded by means of a resolvent observability.

\section{References}

[1] D. S. Almeida Júnior, M. L. Santos and J. E. Muñoz Rivera, Stability to 1-D thermoelastic Timoshenko beam acting on shear force, Z. Angew. Math. Phys. 65 (2014), no. 6, 1233-1249.

[2] M. O. Alves, A. H. Caixeta, M. A. Jorge Silva, J. H. Rodrigues and D. S. Almeida Jnior, On a Timoshenko system with thermal coupling on both the bending moment and the shear force, J. Evol. Equ. 20 (2020), 295-32. 
[3] M. O. Alves, E. H. Gomes Tavares, M. A. Jorge Silva and J. H. Rodrigues, On Modeling and Uniform Stability of a Partially Dissipative Viscoelastic Timoshenko System, SIAM J. Math. Anal. 51 (2019), no. 6, 4520-4543.

[4] M. S. Alves, M. A. Jorge Silva, T. F Ma and J. E. Muñoz Rivera, Invariance of decay rate with respect to boundary conditions in thermoelastic Timoshenko systems, Z. Angew. Math. Phys. (2016) 67: 70.

[5] M. S. Alves, M. A. Jorge Silva, T. F. Ma and J. E. Muñoz Rivera, Non-homogeneous thermoelastic Timoshenko systems, Bull. Braz. Math. Soc. (N.S.) 48 (2017), no. 3, 461-484.

[6] F. Ammar-Khodja, A. Benabdallah, J. E. Muñoz Rivera and R. Racke, Energy decay for Timoshenko systems of memory type, J. Differential Equations 194 (2003) 82-115.

[7] T. A. Apalara, General stability of memory-type thermoelastic Timoshenko beam acting on shear force, Contin. Mech. Thermodyn. 30 (2018), no. 2, 291-300.

[8] C. J. K. Batty, Tauberian theorems for the Laplace-Stieltjes transform. Trans. Amer. Math. Soc. 322 (1990), 783-804

[9] C. J. K. Batty, Asymptotic behaviour of semigroups of operators, In: Functional Analysis and Operator Theory (Warsaw, 1992), vol. 30. Banach Center Publ. Polish Acad. Sci.,Warsaw, 1994.

[10] I. Elishakoff, Who developed the so-called Timoshenko beam theory?, Math. Mech. Solids 25 (2020), 97-116.

[11] L. H. Fatori, R. N. Monteiro and H. D. Fernández Sare, The Timoshenko system with history and Cattaneo law. Applied Mathematics and Computation 228 (2014) 128-140.

[12] B. Feng, Uniform decay of energy for a porous thermoelasticity system with past history, Applicable Anal. 97 (2018), no. 2, 210229.

[13] H.D. Fernández Sare and J.E. Muñoz Rivera, Optimal rates of decay in 2-d thermoelasticity with second sound, J. Math. Phys. 53 (2012), 073509-1-13.

[14] H. D. Fernández Sare and R. Racke, On the stability of damped Timoshenko systems: Cattaneo versus Fourier law, Arch. Rational Mech. Anal. 194 (2009) 221-251.

[15] L. Gearhart, Spectral theory for contraction semigroups on Hilbert space, Trans. Amer. Math. Soc. 236 (1978) 385-394.

[16] M. Grasselli and V. Pata, Uniform attractors of nonautonomous dynamical systems with memory, Progress Nonlinear Differential Equations Applications 50 (2002), 155-178.

[17] F. L. Huang, Characteristic conditions for exponential stability of linear dynamical systems in Hilbert spaces, Ann. Differential Equations 1 (1985) 43-56.

[18] Z. Liu and S. Zheng, Semigroups Associated with Dissipative Systems, Chapman \& Hall/CRC, Boca Raton, 1999.

[19] S. A. Messaoudi and A. Fareh, General decay for a porous thermoelastic system with memory: the case of equal speeds, Nonlinear Anal. 74 (2011), no. 18, 6895-6906. 
[20] S. A. Messaoudi and A. Fareh, General decay for a porous thermoelastic system with memory: the case of nonequal speeds, Acta Math. Sci. Ser. B 33 (2013), no. 1, 23-40.

[21] J. E. Muñoz Rivera and H. D. Fernández Sare, Stability of Timoshenko systems with past history, J. Math. Anal. Appl. 339 (2008), no. 1, 482-502.

[22] J. E. Muñoz Rivera and R. Racke, Mildly dissipative nonlinear Timoshenko systems - global existence and exponential stability, J. Math. Anal. Appl. 276 (2002) 248-278.

[23] R. Quintanilla and R. Racke, Addendum to: Qualitative aspects of solutions in resonators. Arch. Mech. 63 (2011), 429-435.

[24] R. Racke, Heat conduction in elastic systems: Fourier versus Cattaneo, In: Proc. 11th International Conference on Heat Transfer, Fluid Mechanics and Thermodynamics, Skukuza, South Africa (2015), 356-360. EDAS, Leonia, NJ, USA (2015).

[25] M. L. Santos, D. S. Almeida Júnior and J. E. Muñoz Rivera, The stability number of the Timoshenko system with second sound, J. Differential Equations 253 (2012) 2715-2733.

[26] J. Prüss, On the spectrum of $C_{0}$-semigroups, Trans. Amer. Math. Soc. 284 (1984) 847-857.

[27] A. Soufyane, Stabilisation de la poutre de Timoshenko, C. R. Acad. Sci. Paris, Sér. I Math. 328 (1999) 731734.

[28] A. Soufyane, Energy decay for porous-thermo-elasticity systems of memory type, Applicable Anal. 87 (2008), no. 4, 451-464.

[29] S. P. Timoshenko, On the correction for shear of the differential equation for transverse vibrations of prismatic bars, Philosophical Magazine, Series 6, 41, issue 245, (1921) 744746 . 